DOI: https://doi.org/10.15517/rce.v37i2.38868

\title{
EMPAREJAMIENTO ENTRE COMPETENCIAS Y EMPLEO EN EL MERCADO LABORAL COSTARRICENSE
}

\author{
SKILL MISMATCH IN THE COSTA RICAN LABOR MARKET
}

\author{
Laura C. Blanco ${ }^{1}$
}

Recibido: 03/09/2019

Aprobado: 26/11/2019

\begin{abstract}
RESUMEN
Se utilizó la Encuesta Nacional de Hogares 2011-2017 para estimar los tipos de emparejamiento laborales a partir de la definición de Chevalier (2003). Se estudian las covariantes al tipo de emparejamiento utilizando un análisis logit multinomial y se analizan las ecuaciones salariales en función del tipo de emparejamiento y el nivel de competencias. Para la población ocupada mayor de edad, se encuentra una tasa de subcalificación de $28,9 \%$, mientras que las tasas de aparente y genuina sobrecalificación son de $13,3 \%$ y 0,8\%, respectivamente. Los resultados muestran que la subcalificación se encuentra asociada a un premio salarial de aproximadamente $30 \%$, mientras que la aparente sobrecalificación y la sobrecalificación real presentan un castigo promedio equivalente al 35\% y 47,5\% del salario, respectivamente. Para la población con estudios superiores, el castigo a la aparente y genuina sobrecalificación se estiman en $38,9 \%$ y 49,3\%, respectivamente.

PALABRAS CLAVE: : EDUCACIÓN, EDUCACIÓN SUPERIOR, CAPITAL HUMANO, FUNCIONES DE INGRESO DE MINCER, TASAS DE RETORNO, DECISIÓN OCUPACIONAL, COMPETENCIAS, CAPACITACIÓN OCUPACIONAL.

CLASIFICACIÓN JEL: : I21, I23, I26, J24
\end{abstract}

\section{ABSTRACT}

Skill mismatches are estimated using the National Household Survey 2011-2017, as defined by Chevalier (2003). A logit multinomial analysis is used to study the covariates of skill mismatches and wage equations are analyzed as a function of the workers' skill mismatches and competence levels. The working adult population is found to have an underqualification level of $28.9 \%$, while apparent and genuine overqualification levels are $13.3 \%$ and $0.8 \%$, respectively. Results show that underqualification is associated to a wage premium of about $30 \%$, while apparent and genuine overqualification have a $35 \%$ and $47.5 \%$ wage penalty, respectively. Graduates from tertiary institutions with an apparent overqualification level report a wage penalty of $38.9 \%$, and it increases to $49.3 \%$ for those with a genuine overqualification level

KEY WORDS: SCHOOLING, HIGHER EDUCATION, HUMAN CAPITAL, MINCER EARNINGS FUNCTION, RATE OF RETURN, OCCUPATIONAL CHOICE, SKILL, OCCUPATIONAL TRAINING.

JEL CLASIFICATION : I21, I23, I26, J24

1 Universidad de Costa Rica, Escuela de Economía; Código postal 11501-2060; San José, Costa Rica; lauracristina.blanco@ucr.ac.cr 


\section{INTRODUCCION}

El mercado laboral costarricense sufre una serie de problemas estructurales que explican la creciente desigualdad en el país, entre ellos: una alta concentración del empleo formal en pocas empresas, marcadas diferencias salariales entre las actividades productivas y un mayor crecimiento del empleo informal y de mala calidad (Programa Estado de la Nación, 2019). Estos problemas estructurales se manifiestan en indicadores económicos desalentadores, tales como tasas de desempleo superiores al 11\%, tasas de ocupación cercanas al 50\% y una baja participación laboral (cercana al 60\%), así como un nivel de informalidad superior al 46\% para la población ocupada (INEC, 2019).

Detrás de esta dualidad en el mercado laboral, donde un pequeño grupo tiene acceso a empleos formales de calidad mientras la mayoría enfrenta empleos de baja calidad, se halla una considerable disparidad educativa: en promedio, la población en edad de trabajar apenas reporta 8 años de escolaridad, $70 \%$ no concluyó secundaria y apenas 11\% cuenta con estudios universitarios (INEC, 2018). Es de esperar que estas disparidades perpetúen la dualidad observada en el mercado laboral y que una mayoría de personas se inserte a este mercado sin haber desarrollado las habilidades básicas para los puestos de trabajo a los que aspira. Este mal emparejamiento entre las habilidades requeridas por el puesto de trabajo y las poseídas por la persona que ocupa dicho puesto constituye una ineficiencia adicional en el mercado.

En este artículo se estima el porcentaje de la población ocupada mayor de dieciocho años en Costa Rica que se encuentra subcalificada y sobrecalificada, en relación con su puesto de trabajo para el período 2011-2017. El principal resultado es que, efectivamente, existe un considerable porcentaje de la población ocupada que no se encuentra debidamente empatada con su puesto de trabajo. Particularmente, el porcentaje de subcalificación es elevado. Se encuentra que los resultados varían considerablemente según el método de estimación. Utilizando un criterio normativo, se obtiene un porcentaje de subcalificación de 28,9\%, una aparente sobrecalificación de $13,3 \%$ y una sobrecalificación de apenas 0,8\%. Asimismo, los resultados muestran que la subcalificación se encuentra asociada a un premio de aproximadamente $30 \%$ en el ingreso, mientras que la aparente sobrecalificación y la sobrecalificación real presentan un castigo que ronda el 35\% y 47,5\% del ingreso, respectivamente. Estos resultados perfilan un mercado laboral dominado por una oferta de mano de obra con baja calificación, sumándose así a la literatura existente que pone en evidencia las deficiencias del sistema educativo costarricense (OCDE, 2017; Schleicher, 2017) y señalan la necesidad de mejorar las políticas públicas en torno a la educación, para lograr una mejor coordinación de las estrategias de desarrollo.

\section{ANTECEDENTES}

Existe una vasta literatura sobre los rendimientos de la educación utilizando ecuaciones de Mincer (véase, por ejemplo, Blau, 1999; Blau y Kahn, 2007; Chevalier, 2011; European Commission, 2005; Montenegro \& Patrinos, 2013; Mumford y Smith, 2009) que presenta robustez en los resultados: en promedio, los rendimientos de la educación oscilan entre $8 \%$ y $12 \%$. Las mujeres tienden a presentar rendimientos más elevados que los hombres, en parte porque se les exige niveles de productividad más elevados para poder competir, dados los ambientes con presencia de discriminación. Las economías menos desarrolladas también presentan rendimientos superiores que reflejan la escasez de mano de obra calificada y los rendimientos marginales decrecientes asociados a la inversión. Asimismo, los rendimientos de la educación primaria y terciaria tienden a ser superiores a los de la educación secundaria, reflejando las ventajas de la especialización, pues la educación secundaria es más bien una etapa de transición (Montenegro \& Patrinos, 2013). Por 
su parte, la educación técnica vocacional exhibe rendimientos individuales y sociales más elevados que la educación secundaria (Bennell, 1996). Esta, además, produce arraigos más fuertes hacia el mercado laboral y permite capacitar a una población que tiene mayores posibilidades de deserción y menores habilidades para tener éxito en el sistema educativo (Eichhorst, Rodríguez-Planas, Schmidl \& Zimmermann, 2005).

Si bien lo anterior señala la necesidad de contar con educación técnica y universitaria, sus rendimientos resultan también sensibles a las posibilidades de empleo existentes, al depender de la capacidad de las personas para ocupar los puestos vacantes en el mercado laboral (European Commission, 2005). Así, es usual encontrar que un porcentaje de la población se encuentre laborando en puestos de trabajo que no concuerdan con su nivel de capital (emparejamiento vertical) o con su área de entrenamiento (emparejamiento horizontal) (Boll, Leppin, Rossen \& Wolf, 2016). Algunas personas se ubican en puestos de trabajo que exigen un nivel educativo superior al que ellas poseen (subcalificación), lo que les obliga a capacitarse en el trabajo o a exhibir productividades laborales inferiores a quienes sí cuentan con las calificaciones requeridas. En contraste, quienes no encuentran trabajo podrían aceptar puestos con requerimientos inferiores a los correspondientes con su nivel educativo (sobrecalificación).

Freeman (1976, citado en Ortiz \& Kucel, 2008; Kucel, 2011, Chevalier, \& Lindley, 2009) es quien primero analiza el problema de la sobreeducación universitaria y propone que esta obedece a una imperfección temporal del mercado laboral: el exceso de oferta de personas universitarias generaría una disminución de los retornos de la educación. Esto, a su vez, reduciría el número de personas graduadas de la universidad, lo que terminaría por reestablecer el equilibrio de mercado. No obstante, los retornos a la educación no han disminuido a lo largo del tiempo, en parte por la creciente demanda por mano de obra calificada y al desarrollo tecnológico (Quintini, 2011), así como por el creciente requerimiento de habilidades para un mismo puesto de trabajo, debido a las tareas más demandantes asociadas a la ocupación (Gallie \& White, 1993, citado en Alpin, Shackleton, \& Walsh, 1998).

La incidencia de la sobreeducación lejos de ser un fenómeno transitorio, tal y como lo predecía Freeman, parece ser permanente. En el Reino Unido, por ejemplo, se estima que los malos emparejamientos afectan a alrededor de la mitad de las personas graduadas de educación técnica y universidades (Allen \& van der Velden, 2001) y al 38\% de la población ocupada (Sutherland, 2012); entre el 15\% (Dolton \& Silles, 2001) y 30\% (Battu, Belfield, \& Sloane, 1999) de las personas graduadas de universidades nunca llega a tener un trabajo que requiera de un título universitario y encontrarse en un puesto para el cual se tiene una sobrecalificación aumenta la probabilidad de volver a encontrarse en esta condición en el siguiente trabajo (Dolton \& Silles, 2001). Además, la sobrecalificación se concentra en personas con bajos niveles de habilidades (Groot \& van den Brink, 2000). Las tasas de sobreeducación también varían entre países. En Estados Unidos, las tasas de sobreeducación para la población con título universitario fluctúan entre 15\% y 40\%; en Bélgica, son alrededor del 50\% (Kucel, 2011); en Paquistán, 30\% (Farooq, 2011); en España, 17\% (Alba-Ramírez, 1993); y en Australia, se estima que el 11,5\% de la población ocupada se encuentra sobrecalificada (Mavromaras, McGuinness \& Fok, 2009). En general, el promedio de sobreeducación asciende a 23,3\% y el de la subeducación, a 14,4\% (Groot \& van den Brink, 2000), aunque en países como España o Bélgica, esta última puede superar el 20\% (Alba-Ramírez, 1993 y ILO, 2014). En algunos lugares, tales como Finlandia, la subeducación incluso puede ser un problema más recurrente que la sobreeducación (Jauhiainen, 2011).

Estos malos emparejamientos introducen ineficiencias en el mercado laboral y afectan en mayor medida a las personas sobrecalificadas (Boll, Leppin, Rossen \& Wolf, 2016). Estas tienen rendimientos de la educación inferiores a sus pares en trabajos empatados con sus habilidades (Alpin, Shackleton, \& Walsh, 1998; Verhaest \& Omey, 2006), i.e., en general, ganan menos que personas con su mismo nivel de calificación que se encuentra en un trabajo acorde con esta, 
aunque más que las personas en el mismo puesto de trabajo, cuyo nivel de calificación concuerda con el requerido para el trabajo (Sloane, Battu, \& Seaman, 1999; Quintini, 2011). Además, participan menos de capacitaciones laborales que quienes poseen su mismo nivel de calificación (Alpin, Shackleton, \& Walsh, 1998; Verhaest \& Omey, 2006; Quintini, 2011), reportan niveles de satisfacción laboral por debajo de sus pares (Alpin, Shackleton, \& Walsh, 1998; Allen \& van der Velden, 2001; Verhaest \& Omey, 2006; Quintini, 2011), menores productividades (Verhaest \& Omey, 2006; Quintini, 2011), menores ascensos y experiencia como supervisores (Sloane, Battu, \& Seaman, 1999; McMillen, Seaman, \& Singell Jr., 2007), mayor ausentismo (Alpin, Shackleton, \& Walsh, 1998), rotación en el trabajo (Alba-Ramírez, 1993; Verhaest \& Omey, 2006; Quintini, 2011), despidos y desempleo debido a su menor calidad laboral (Sloane, Battu, \& Seaman, 1999) y mayor obsolescencia de habilidades (Dolton \& Silles, 2001) y deterioro cognitivo (de Grip, Bosma, Willems, \& van Boxtel, 2008); además de salarios de largo plazo, riqueza e incluso clase social inferiores a las de sus pares (Kucel, 2011).

Por su parte, las personas subcalificadas ganan más y participan más de capacitaciones que las personas con su mismo nivel de calificación que se encuentran bien empatadas, pero menos que las personas en su puesto de trabajo que cuentan con la calificación requerida para el puesto (Sloane, Battu, \& Seaman, 1999, Verhaest \& Omey, 2006, Quintini, 2011). Reportan, además, menor deterioro cognitivo debido a que se encuentran con mayores retos laborales (de Grip, Bosma, Willems, \& van Boxtel, 2008). La subcalificación no parece tener impacto sobre otras variables, como la satisfacción o movilidad laboral (Verhaest \& Omey, 2006).

Existen seis teorías desde las cuales puede explicarse el problema de los emparejamientos laborales $^{2}$. La primera de ellas es la teoría del capital humano (Becker, 1962, 1993, Schultz, 1974 y Mincer, 1974), i.e., el acervo de conocimientos, habilidades y destrezas productivas de una persona (Becker, 1962). El proceso de acumulación en capital humano supone un análisis dinámico costobeneficio en donde se valoran las ganancias en el tiempo asociadas a la inversión en capital humano contra los costos de la inversión. Los malos emparejamientos laborales serían temporales, ya que las habilidades y el nivel de productividad se ajustarían endógenamente. Aunque sí recibe alguna validación debido a la robustez con que la experiencia afecta negativamente la probabilidad de sobrecalificación, dicha teoría no puede explicar la persistencia de los malos emparejamientos (Boll, Leppin, Rossen \& Wolf, 2016).

La segunda es la teoría de la movilidad laboral u ocupacional, según la cual, una parte de los rendimientos de la educación consiste en incrementar las probabilidades de ascenso (Sicherman \& Galor, 1990). Las personas entran al mercado laboral a puestos para los cuales se encuentran sobrecalificadas con la expectativa de ganar experiencia y ser ascendidas en el futuro, explicando así la mayor concentración de personas jóvenes dentro de la población sobrecalificada (Sicherman, 1991). Igualmente, las personas subcalificadas serían aquellas que, habiendo demostrado mayores habilidades, fueron entrenadas en el trabajo y, posteriormente, ascendidas (McMillen, Seaman, \& Singell Jr., 2007). La evidencia empírica no apoya esta teoría (Boll, Leppin, Rossen \& Wolf, 2016), aunque Alba-Ramírez (1993) sí encuentra evidencia a favor de ella. Al igual que la teoría de capital humano, se le cuestiona que, bajo esta explicación, la sobrecalificación constituiría un problema temporal de oferta de la mano de obra (Quintini, 2011), que se corregiría mediante el ascenso laboral o la movilidad fuera de la empresa (Ortiz \& Kucel, 2008).

2 Si bien este trabajo no pretende falsear las diferentes teorías que explican la existencia de la sub y sobrecalificación, se considera importante exponerlas. En este sentido, el trabajo no se enmarca en ninguna teoría particular para explicar las causas del fenómeno, sino que se limita a cuantificarlo. Similarmente, la mayoría de trabajos empíricos se limitan a cuantificar el nivel de sub y sobrecalificación y las variables correlacionadas, sin buscar falsear una u otra teoría. Quienes sí se han propuesto hacer esto, han encontrado evidencia a favor sobre todo de la teoría de la asignación (Allen \& van der Velden, 2001; Quintini, 2011; Boll, Leppin, Rossen \& Wolf, 2016), aunque Alba-Ramírez también encuentra evidencia a favor de la movilidad laboral y el emparejamiento (Alba-Ramírez, 1993). 
Por el contrario, la teoría sobre la competencia de trabajos propone que el mercado laboral no es un espacio donde se subasten habilidades entre oferentes y demandantes, sino más bien una oferta de puestos de entrenamiento, i.e., dado que la mayoría de las habilidades se desarrollan en el trabajo, los puestos vacantes se asignan conforme a una lógica de eficiencia sobre el costo de entrenamiento de la persona que lo ocupará. Las personas, entonces, tendrán un incentivo a sobrecalificarse, pues esto conlleva un menor costo de entrenamiento que les permite saltar al inicio de la fila de empleo (Thurow, 1975). Así, las personas trabajadoras compiten por lugares de trabajo sujetos a sus calificaciones (Quintini, 2011; Boll, Leppin, Rossen \& Wolf, 2016), tal que la sobrecalificación es una característica estructural del mercado laboral (Ortiz \& Kucel, 2008), que evidencia la capacidad de entrenamiento de la persona (Alpin, Shackleton, \& Walsh, 1998).

En forma similar, la teoría de la señalización (Spence, 1973) supone que el mercado laboral es imperfecto, por tanto, es necesario señalizar las habilidades durante el proceso de búsqueda de trabajo. Dado que las personas menos hábiles tienen un mayor costo de capacitación, contar con una calificación terciaria señaliza que la persona es de alta productividad. Si el costo de estudiar en la universidad baja, personas menos hábiles podrán ser capaces de obtener dichas calificaciones, dificultándole a las empresas identificar a aquellas más hábiles. Esto genera presión para que las personas obtengan aún títulos más altos, empujando al alza la inflación de las calificaciones y la sobrecalificación (Sloane, Battu, \& Seaman, 1999; Dolton \& Silles, 2001; Quintini, 2011). Luego, se perpetúa una polarización del mercado laboral, donde las personas más hábiles se colocan en empleos de alto nivel, pero un grupo importante de personas con calificaciones altas debe colarse en puestos menores. Al igual que la teoría de competencia laboral, en la teoría de la señalización, las personas sobrecalificadas esperarían recibir capacitaciones y ascensos más rápido, así como un mayor crecimiento salarial, usualmente entre diferentes segmentos de mercado, mientras que las personas subcalificadas recibirían ascensos graduales dentro de su propio segmento laboral (McMillen, Seaman, \& Singell Jr., 2007).

En quinto lugar, la teoría del emparejamiento laboral propone que las personas reciben como salario el equivalente de su productividad marginal, condicionada a la información disponible. Por tanto, las personas tienen un incentivo a mantenerse en trabajos donde su productividad es relativamente alta (Jovanovic, 1979), pero se mantendrán alertas a "comprar trabajos" que les permita moverse a mejores emparejamientos conforme la información mejore (Alba-Ramírez, 1993). Esto supone que las personas tenderán a moverse temprano en su vida laboral, pues los malos emparejamientos serán detectados poco después de que la persona sea contratada y serán más eficientes conforme aumente su experiencia y entrenamiento en el trabajo. Alba-Ramírez (1993) también encuentra evidencia que apoya esta teoría, pues las personas sobrecalificadas tienden a cambiar más frecuentemente de trabajo y a tener una menor duración en él debido a que se encuentran permanentemente buscando trabajo.

Por último, la teoría de la asignación propone que los salarios cumplen una función de asignación de personas trabajadoras entre sectores, quienes son atraídas a ese sector con la expectativa de que su decisión maximice sus ingresos futuros (Sattinger, 1993). Dados los costos de búsqueda, una persona podría optar por un puesto para el cual está sobrecalificada, lo que constituye una ineficiencia del proceso de asignación. Esto, a su vez, limitaría la productividad de la persona, al ajustarse a la menor complejidad de su trabajo (Quintini, 2011), lo que resultaría en un menor ingreso (Ortiz \& Kucel, 2008). Esta teoría es particularmente útil para explicar la mayor sobrecalificación de mujeres: dado que las mujeres casadas priorizan la ubicación del trabajo de su esposo, sus costos de búsqueda son mayores al tener que restringir sus posibilidades laborales a las limitaciones geográficas de su pareja (Boll, Leppin, Rossen \& Wolf, 2016). Esta es la teoría que muestra la mayor consistencia con la evidencia empírica (Allen \& van der Velden, 2001; Quintini, 2011; y Boll, Leppin, Rossen \& Wolf, 2016). 
Por su parte, la cuantificación de la sobrecalificación (sobreeducación) se realiza mediante tres métodos: el objetivo o normativo, el subjetivo y el estadístico (Alpin, Shackleton, \& Walsh, 1998; Dolton \& Silles, 2001; Quintini, 2011). En el primero, se asocian los niveles de ocupaciones a niveles educativos (años de escolaridad), utilizando las escalas de clasificación de ocupaciones o mediante la visita de una persona que analiza el puesto de trabajo, y se clasifica como sub-calificada (subeducada) a aquella persona que tenga un nivel educativo (años de escolaridad) inferior al que corresponde. En forma similar, se considera sobrecalificada (sobreeducada) a aquella persona que tenga un nivel educativo (años de escolaridad) superior al establecido. El método normativo tiende a estimar mayores tasas de sobrecalificación y sobreeducación (Dolton \& Silles, 2001). El método subjetivo consiste en preguntarle a la persona entrevistada si su nivel de calificación (años de escolaridad) coincide con el requerido por su puesto de trabajo y resulta relativamente fácil en cuanto a la recolección de la información (Sloane, Battu, \& Seaman, 1999). Sin embargo, es el método que genera las mayores estimaciones de sobrecalificación (Groot \& van den Brink, 2000). El método estadístico calcula los años de escolaridad promedio (Verdugo \& Verdugo, 1988; ILO, 2014) o nivel de instrucción modal (Boll, Leppin, Rossen \& Wolf, 2016) y califica como malos emparejamientos a quienes se desvíen en una distancia específica de este valor, usualmente una desviación estándar. En otras ocasiones se define un percentil particular a partir del cual la persona se considera sobrecalificada (Ortiz \& Kucel, 2008). Este método tiene la debilidad de que, al usar la desviación estándar sobre distribuciones que tienden a ser normales, estima menores tasas de sobrecalificación (Groot \& van den Brink, 2000; Jauhiainen, 2011; ILO, 2014); sin embargo, una fortaleza es que podría reflejar cambios en los requerimientos para puestos de trabajo a lo largo del tiempo al permitir observar la evolución de la media en años de escolaridad para los diferentes puestos de trabajo (ILO, 2014). En general, los resultados obtenidos pueden diferir significativamente según el método de estimación (Groot \& van den Brink, 2000; Verhaest \& Omey, 2006; Farooq, 2015).

Por su parte, Chevalier (2003) argumenta sobre la necesidad de distinguir entre aquellas personas que se encuentran genuinamente sobrecalificadas y quienes solo lo están en apariencia. Para ello, introduce en el modelo como variables proxy de las habilidades el nivel de satisfacción en el trabajo. Quienes estén genuinamente sobrecalificadas, son personas que estarán disconformes con su trabajo actual, pues este no empata con sus habilidades. Por el contrario, quienes hayan sobre invertido en educación, pero tengan productividades bajas, se encontrarán a gusto en el trabajo, pues este sí es compatible con sus verdaderas habilidades. Tanto Chevalier (2003) como Chevalier y Lindley (2009) estiman que la población sobreeducada ronda entre el 30\% y 35\% de las personas graduadas de universidad y ocupadas en Reino Unido. De estas, cerca del $20 \%$ en realidad se encuentran aparentemente sobreeducadas y $15 \%$ genuinamente sobreeducadas (Chevalier \& Lindley, 2009). Este último grupo, a pesar de requerir menos tiempo en capacitación, es más propenso a carecer de las habilidades relacionadas con la universidad. En contraste, las personas aparentemente sobreeducadas son más proclives a tomar un trabajo por interés y a laborar en un puesto que no requiere título universitario o de una ocupación que apenas recientemente empezó a exigir un título universitario (Chevalier \& Lindley, 2009). Chevalier (2003) también encuentra que la aparente sobreeducación se penaliza con una reducción del ingreso entre 5\% y 11\% y la sobreeducación genuina, entre 22 y $26 \%$ relativo a sus pares. Similares resultados se obtienen en Chevalier y Lindley (2009).

Empíricamente, se ha identificado algunos de los determinantes de la sobrecalificación. Así, las personas con programas de estudio más largos (Battu, Belfield, \& Sloane, 1999) y con mejores notas (Chevalier, \& Lindley, 2009; Dolton \& Silles, 2001; Marqués Perales, \& Gil-Hernández, 2015) tienen una mejor probabilidad de encontrar trabajos acordes con su nivel de calificación, mientras que quienes estudiaron en edad madura presentan una menor probabilidad de encontrar un trabajo adecuado (Battu, Belfield, \& Sloane, 1999). Graduarse de una universidad nueva, 
usualmente asociada a carreras no tradicionales, incrementa la probabilidad de ser aparentemente sobreeducada, mientras que obtener un posgrado (Dolton \& Silles, 2001; Chevalier, \& Lindley, 2009) y contar con habilidades profesionales, tales como liderazgo, reduce la probabilidad de una genuina sobreeducación, pero tener progenitores en condición de desempleo la aumenta (Chevalier, \& Lindley, 2009).

Escoger una disciplina de estudio con barreras de entrada por un alto requerimiento de habilidades (Boll, Leppin, Rossen \& Wolf, 2016), con mayor especialización técnico-científica (Marqués Perales, \& Gil-Hernández, 2015) o con una de mayor demanda reduce la probabilidad de sobreeducación, en particular matemática (Chevalier, 2003; Chevalier, \& Lindley, 2009), mientras que carreras como biología, agricultura, idiomas o humanidades la aumentan (Dolton \& Silles, 2001; Chevalier, 2003). Estas diferencias varían entre países: entre más estratificado y menos estandarizado sea el sistema educativo, mayor serán las diferencias de sobreeducación observadas entre disciplinas de estudio. Fuertes sistemas vocacionales como los de Alemania resultan más eficientes en coordinar adecuadamente las políticas educativas con el mercado laboral que sistemas como los españoles (Ortiz \& Kucel, 2008). En cuanto a las ocupaciones, laborar en transporte, comunicaciones, agricultura, hoteles y restaurantes aumenta la probabilidad de sobreeducación (Alpin, Shackleton, \& Walsh, 1998), mientras que tener un puesto gerencial o profesional la disminuye (Battu, Belfield, \& Sloane, 1999).

La experiencia laboral muestra resultados mixtos con respecto a la sobreeducación: en algunos casos se ha encontrado que esta disminuye la probabilidad de sobreeducación y aumenta la de subeducación (Alba-Ramírez, 1993; Boll, Leppin, Rossen \& Wolf, 2016; Sloane, Battu, \& Seaman, 1999), mientras que en otros no tiene ninguna influencia (Chevalier, \& Lindley, 2009; Dolton \& Silles, 2001) e, incluso, las posibilidades de ascenso a un puesto pueden estar más influidas por el conocimiento interno de la empresa o las capacitaciones recibidas que por la experiencia misma (McMillen, Seaman, \& Singell Jr., 2007). En contraste, existe una robusta evidencia de que la sobrecalificación disminuye con la edad, porque las personas logran modificar sus decisiones de trabajo o capacitación para hacerlas calzar, en tanto que las personas de mayor edad tienden a estar más subcalificadas porque han sido capacitadas y ascendidas en sus lugares de trabajo ${ }^{3}$ (Alba-Ramírez, 1993; Alpin, Shackleton, \& Walsh, 1998; Chevalier, 2003; ILO, 2014; Quintini, 2011; Sutherland, 2012). Sin embargo, la probabilidad de sobrecalificación vuelve a incrementarse después de la edad de retiro (Boll, Leppin, Rossen \& Wolf, 2016).

La sobrecalificación también se asocia con las características del lugar de trabajo: contar con un trabajo interino o temporal aumenta la probabilidad de sobrecalificación (Alpin, Shackleton, \& Walsh, 1998; Boll, Leppin, Rossen \& Wolf, 2016; Dolton \& Silles, 2001; Sloane, Battu, \& Seaman, 1999; Sutherland, 2012), en tanto la antigüedad laboral y el tamaño de la empresa la disminuyen (Alba-Ramírez, 1993; Battu, Belfield, \& Sloane, 1999; Boll, Leppin, Rossen \& Wolf, 2016; Dolton \& Silles, 2001; Sloane, Battu, \& Seaman, 1999; Sutherland, 2012; Ortiz \& Kucel, 2008; Quintini, 2011). Los malos emparejamientos son más frecuentes en el sector privado debido a que el sector público tiende a tener escalas salariales y de contratación más homogéneas (Alpin, Shackleton, \& Walsh, 1998; Dolton \& Silles, 2001; Sutherland, 2012). Estos también son más frecuentes cuando aumenta la tasa de crecimiento de la fuerza laboral, debido a que la mayor oferta de mano de obra presiona por una mayor demanda de calificaciones (Groot \& van den Brink, 2000), y cuando hay presencia de sindicatos, debido a que estos imponen sistemas de contratación y remuneración que no necesariamente responden a las habilidades y el desempeño laboral (Quintini, 2011). Por el contrario, si el mercado laboral es flexible (Battu, Belfield, \& Sloane, 1999) y cuenta con regulaciones de empleo y competencia internacional (Quintini, 2011), los emparejamientos adecuados tienden a aumentar.

3 Esto podría identificarse como evidencia a favor de la teoría de la movilidad laboral, pues la sobreeducación se concentraría en personas jóvenes y la subeducación en personas de mayor edad. 
Los malos emparejamientos también son menos frecuentes en ciudades grandes, metropolitanas o céntricas o cuando las personas tienen facilidades de desplazamiento, porque esto les permite un espectro de búsqueda de trabajo más acorde con sus habilidades (Jauhiainen, 2011). Lo mismo ocurre para aquellas personas dispuestas a reubicarse por su trabajo dentro de su país (Dolton \& Silles, 2001), mas no así para migrantes (Boll, Leppin, Rossen \& Wolf, 2016; Fernández \& Ortega, 2006; Quintini, 2011) ni minorías étnicas (Alpin, Shackleton, \& Walsh, 1998; Chevalier, 2003), para quienes la probabilidad de sobreeducación más bien aumenta. Similarmente, vivir en una zona rural, particularmente si se es mujer, supone un mayor riesgo de sobreeducación (Boll, Leppin, Rossen \& Wolf, 2016). Y, en general, ser mujer se asocia con un mayor (menor) riesgo de sobreeducación (subeducación) (Alpin, Shackleton, \& Walsh, 1998, Battu, Belfield, \& Sloane, 1999, Chevalier, 2003; Farooq, 2011; 2015; Groot \& van den Brink, 2000; ILO, 2014; Jauhiainen, 2011; Sutherland, 2012). Esto pareciera afectar particularmente a las mujeres casadas, quienes dependen en mayor medida del tamaño del mercado laboral, al tener mayores restricciones para desplazarse hacia su lugar de trabajo 4 (Jauhiainen, 2011). No obstante, otros estudios no encuentran ningún efecto del estado civil sobre la probabilidad de las mujeres de ser sobreeducadas (Dolton \& Silles, 2001) y en otras ocasiones, más bien, se ha encontrado que son los hombres casados quienes ven incrementada su probabilidad de ser subeducados (Sloane, Battu, \& Seaman, 1999).

Tampoco existe evidencia contundente sobre cómo la estructura familiar puede influir sobre la probabilidad de sobreeducación: en algunos casos, se ha encontrado que contar con menores incide positivamente sobre la probabilidad de una mujer de ser sobreeducada, mas no así sobre la del hombre (Boll, Leppin, Rossen \& Wolf, 2016; Jauhiainen, 2011; Sloane, Battu, \& Seaman, 1999); en otros, más bien la tenencia de menores de edad disminuye la probabilidad de sobreeducación al presionar a sus progenitores a conseguir los mejores trabajos disponibles (Dolton \& Silles, 2001). Las probabilidades de lograr un buen emparejamiento laboral dependen también del contexto familiar: la presencia de otras personas adultas desempleadas o dependientes (Boll, Leppin, Rossen \& Wolf, 2016) y pertenecer a hogares con deuda financiera (Dolton \& Silles, 2001) incrementan la probabilidad de sobreeducación, mientras que provenir de un hogar acomodado la disminuye (Marqués Perales, \& Gil-Hernández, 2015; Ortiz \& Kucel, 2008).

Tal y como se mencionó, los malos emparejamientos producen efectos sobre diversas variables socioeconómicas, incluido el ingreso. Al comparar, los salarios relativos a personas con el mismo nivel de calificación o educación se encuentra un premio a la subcalificación o subeducación y un castigo a la sobrecalificación o sobreeducación (Chevalier, 2003; Chevalier, \& Lindley, 2009; Dolton \& Silles, 2001; Verdugo \& Verdugo, 1988; Verhaest \& Omey, 2006), lo que sugiere que las personas sobreeducadas se ubican en puestos más bajos o de menor productividad. La penalización a la sobreeducación oscila entre 5\% en Estados Unidos (Verdugo \& Verdugo, 1988) y 53\% en Paquistán (Farooq, 2015), mientras que el premio a la subcalificación puede ascender hasta a 12,5\% en España (Alba-Ramírez, 1993). Por su parte, si se comparan los salarios relativos a los requerimientos de calificación o educación exigidos por el puesto de trabajo, se encuentra que existe una ganancia a sobreeducarse o sobrecalificarse y una penalización por subeducarse o subcalificarse respecto al requerimiento laboral, que rondan cerca del 5\% en cada caso (AlbaRamírez, 1993; Battu, Belfield, \& Sloane, 1999). Estas estimaciones salariales, además, exhiben robustez respecto a otras variables ya conocidas en la determinación de ecuaciones de Mincer (1974) y sistematizada en Schultz (1974). Por ejemplo, se mantiene que ser mujer (Dolton \& Silles, 2001; Farooq, 2015), pertenecer a una minoría étnica (Verdugo \& Verdugo, 1988) o laborar en el sector público (Farooq, 2015), se asocia a menores ingresos. En contraste, poseer grados académicos universitarios o de posgrado (Allen \& van der Velden, 2001), recibir capacitación en el trabajo (Alba- 
Ramírez, 1993), laborar para una empresa grande (Dolton \& Silles, 2001; Montenegro \& Patrinos, 2013), tener antigüedad laboral (Farooq, 2015) o ser hombre casado (Verdugo \& Verdugo, 1988) se relacionan positivamente al ingreso. Finalmente, el tipo de educación recibida también incide sobre el ingreso: tener una ocupación gerencial, profesional o en educación (Dolton \& Silles, 2001), haber estudiado en el exterior o una universidad prestigiosa (Farooq, 2015) se asocian con mayores salarios, mientras que haber estudiado artes o humanidades (Dolton \& Silles, 2001) se asocia con un menor ingreso.

\section{DATOS Y MÉTODOS}

Los tipos de emparejamiento se cuantifican para la población ocupada mayor de dieciocho años en el mercado laboral costarricense, utilizando la Encuesta Nacional de Hogares (ENAHO), para el período 2011-2017 (INEC, 2018), con los códigos de ocupaciones COCR-2011 (INEC, 2014) desglosados a cuatro dígitos. Se escogió este período debido a que, a partir de 2011, hubo un cambio en la escala de clasificación de ocupaciones. Tras depurar la base de datos y eliminar valores inconsistentes o faltantes, se obtiene una muestra balanceada para la población ocupada mayor de edad de 155646 observaciones, de las cuales 55,8\% corresponden a mujeres y una submuestra de la población ocupada y graduada de estudios superiores de 19329 observaciones, de las cuales 58,4\% son mujeres. Posteriormente, se estimó el tipo de emparejamiento mediante tres métodos diferentes:

1. Método normativo: la Clasificación Internacional Uniforme de Ocupaciones (ILO, 2012a) asigna a cada ocupación su correspondiente nivel de competencias a partir de la escala de competencias ISCO-08 (ILO, 2012b). Esta es una escala con cuatro niveles:

- El primero constituye el nivel de competencias más básico e incluye tareas simples $y$ rutinarias en escritura, lectura y números para las cuales se solicita el ciclo completo de educación primaria o el primer ciclo de la educación básica. En este nivel de competencias se concentran principalmente tareas manuales o físicas e incluye ocupaciones elementales, tales como limpieza o acarreo de materiales.

- El segundo nivel de competencias supone la capacidad de leer información, instrucciones, registrar tareas en forma escrita y un amplio manejo de letras, operaciones $y$ habilidades comunicativas. Para ello, usualmente se exige haber cursado el primer ciclo completo de la educación secundaria o haber concluido el segundo ciclo. Algunas de sus ocupaciones son secretariado, contabilidad, sastrería, policía, mecánica automotriz y electricistas.

- El tercer nivel de competencias exige el desempeño de tareas técnicas y complejas de un área especializada con un elevado conocimiento de letras, matemáticas y comunicación que requiere estudios terciarios por un período de 1 a 3 años después de concluida la secundaria. Algunas ocupaciones de este nivel son la gerencia de comercios, puestos técnicos de laboratorios médicos, de informática, de radiodifusión y de representación comercial.

- El cuarto nivel de competencias supone habilidades en solución de problemas complejos y la adopción de decisiones, así como un amplio conocimiento teórico y fáctico en una especialización particular. Estas habilidades se adquieren después de haber cursado, al menos, estudios universitarios entre 3 y 6 años y haber obtenido un título. Este nivel incluye, por tanto, a las personas profesionales. 
A partir de esta clasificación y utilizando los años de escolaridad y el último grado obtenido, se asignó un nivel de competencia a cada persona trabajadora. Quienes no hubiesen concluido el nivel de educación general básica (i.e., noveno año) se clasificaron en el nivel 1. Quienes concluyeron la educación general básica o secundaria se clasificaron en el nivel 2. Esto incluye a quienes cursaron estudios técnicos durante secundaria y no continuaron con estudios postsecundarios, ya que el nivel de competencias 3 exige estudios superiores y los diplomados técnicos que se ofrecen en la secundaria costarricense refieren a ocupaciones que se ubican en el nivel de competencias 2, tales como mecánica automotriz, secretariado o turismo. El nivel 3 engloba a aquellas personas que reportan un título superior de 3 años o menos, esto incluye diplomados, estudios parauniversitarios y algunos programas universitarios cortos. Finalmente, quienes se hayan graduado de programas universitarios mayores a tres años se clasificaron en el nivel de competencias 4. Posteriormente, se compara el nivel de competencia de la persona con el requerido por el puesto según ISCO-08. Si la persona tiene un nivel de competencia superior al requerido, se le clasifica como sobrecalificada. Si es inferior, estará subcalificada.

2. Método estadístico utilizando el nivel de competencias: en esta segunda estimación no se le asigna a la ocupación el nivel de competencia establecido en ISCO-08, sino el nivel de competencia modal observado en la muestra. Si el nivel de competencias de la persona supera (es inferior) al de la moda, esta se clasifica como sobrecalificada (subcalificada).

3. Método estadístico utilizando los años de escolaridad: se toma los años de educación promedio de la muestra como el nivel educativo requerido para el puesto y se procede a compararlo con los años de educación reportados por la persona. Si sus años de educación superan al promedio de su ocupación en más de una desviación estándar, la persona se clasifica como sobreeducada. Por el contrario, si estos son menores a la media en más de una desviación estándar, se clasifica como subeducada.

Posteriormente y siguiendo a Chevalier (2003), se descompuso el grupo de personas sobrecalificadas (estimaciones 1 y 2 ) o sobreeducadas (estimación 3) en aparentemente sobrecalificadas (sobreeducadas) y genuinamente sobrecalificadas (sobreeducadas). Según Chevalier (2003), una persona genuinamente sobrecalificada reportará insatisfacción laboral y querrá cambiar de trabajo, por lo que estas variables sirven de proxies para medir la habilidad de la persona relativa a su trabajo. La ENAHO incluye dos preguntas claves para identificar esta situación: una que identifica si se desea cambiar de trabajo y las razones de esto y otra que pregunta por la satisfacción laboral. Así, quienes posean sobrecalificación y además reporten querer cambiar de trabajo debido a que no aprovecha el conocimiento o estudios que tiene o debido a que no puede adquirir o aumentar la experiencia en lo que quiere, así como quienes cuentan con sobrecalificación y reportan insatisfacción laboral por desaprovechamiento de competencias se catalogan con una genuina sobrecalificación o sobreeducación, según sea el caso.

Con el fin de determinar aquellas variables correlacionadas con el tipo de emparejamiento, se estima un modelo logit multinomial (Alba-Ramírez, 1993; Chevalier, 2003; Sloane, Battu, \& Seaman, 1999), aunque otros autores también lo hacen utilizando modelos probit ordenados (McMillen, Seaman, \& Singell Jr., 2007). En este caso y siguiendo a Chevalier (2003), el tipo de emparejamiento (E) depende de una serie de logros educativos y características individuales (representados por la matriz X) y un término normalmente distribuido $\eta$ de componentes no observados, tal que:

$$
E^{*}=\beta X+\eta
$$


Debido a que este modelo latente no puede estimarse, se realiza una estimación logit multinomial a partir de la observación de la variable categórica que identifica el tipo de emparejamiento. Así, se tendrá:

$$
E=\left\{\begin{array}{l}
0 \text { si } E^{*}<\mu_{0} \\
1 \text { si } E^{*}<\mu_{1} \\
2 \operatorname{si} E^{*}<\mu_{2} \\
3 \text { si } E^{*}<\mu_{3}
\end{array}\right.
$$

Es decir, se utiliza una función logit multinomial para identificar a aquellas personas que se encuentran subcalificadas (categoría 0), aparentemente sobrecalificadas (categoría 2) y genuinamente sobrecalificadas (categoría 3), tomando como grupo de referencia a quienes se encuentran adecuadamente empatadas en su trabajo (categoría 1). Además, dentro de las variables de control contenidas en (i) se incluye el inverso de la razón de Mills de la probabilidad de trabajar, con el objetivo de corregir cualquier sesgo de autoselección de la población ocupada (Heckman, 1976) ${ }^{5}$.

Finalmente, se estimaron ecuaciones de Mincer (1974), tanto para la población ocupada como para una submuestra compuesta por la población con estudios terciarios. Las regresiones se calcularon para cada año individualmente y para mujeres $y$ hombres en forma separada. Para ello, se estima una regresión de mínimos cuadrados ordinarios para el logaritmo del salario por hora $(w)$ en función del tipo de emparejamiento $\left(E^{*}\right)$ y una matriz de variables individuales $(Z)$ asociadas al capital humano, al tipo de empleo y características sociodemográficas. Las variables de capital humano incluyen una variable categórica indicando el nivel de competencias, la experiencia laboral y su cuadrado y una variable dicotómica que indica si la persona es bilingüe. Para la submuestra de la población con estudios postsecundarios, se incluye, además, el área de estudio. Nótese que, debido a que la especificación del modelo incluye el nivel de competencia de la persona y no el nivel de competencia requerido, se espera obtener un premio a la subcalificación y un castigo a la sobrecalificación. Las variables relacionadas al empleo incluyen variables dicotómicas para indicar si la persona cuenta con seguro, tiene un trabajo permanente, tiene un trabajo parcial o con una jornada inferior a 40 horas semanales, labora en el sector privado, labora en hogar privado y una variable categórica para el tamaño de la empresa. Por último, las variables sociodemográficas incluyen variables dicotómicas para indicar si la persona es cabeza del hogar, tiene pareja, es migrante, es mujer y si vive en zona urbana, así como una variable categórica indicando la región de residencia.

Ahora bien, tanto Dolton y Silles (2001), Chevalier (2003) y Chevalier y Lindley (2009) advierten de un posible problema de endogeneidad entre el ingreso y el tipo de emparejamiento, ya que estas ecuaciones se determinan simultáneamente. El resultado de esto es que el efecto negativo de la sobreeducación tiende a subestimarse (Dolton y Silles, 2001), reflejando un sesgo de habilidad. En estudios longitudinales (Chevalier, 2003; Chevalier \& Lindley, 2009), esta endogeneidad es corregida mediante la inclusión del error asociado al primer salario -y que sirve como proxy del nivel de habilidad no observado de la persona- en la estimación tanto de la probabilidad de que la persona esté sobreeducada para su segundo trabajo como en la estimación del ingreso del segundo

5 Para esto, se estimaron ecuaciones probit para la probabilidad de empleo en función de los años de experiencia laboral potencial y su cuadrado, el nivel de competencias, la región y zona de residencia, ser bilingüe, cabeza de hogar, migrante, tener pareja o discapacidad. Por razones de espacio, estas ecuaciones no se discuten, pero pueden facilitarse a solicitud. 
trabajo. Debido a que no se cuenta con información longitudinal, se usará la corrección propuesta por Dolton y Silles (2001), la cual consiste en una extensión de Heckman (1976), de forma que en la ecuación de salarios se incluye el término de ajuste $\lambda$,i.e., la inversa de la razón de Mill que se obtiene de la ecuación probit para la sobreeducación junto con los valores observados de sobreeducación. En este caso, dado que se debe estimar una logit multinomial, se incluye tres factores de ajuste $\lambda_{i}$, uno asociado a cada una de las ecuaciones del modelo logit multinomial ${ }^{6}$.

Si $\lambda$ representa la matriz de factores de ajuste, la ecuación de salarios estará dada por:

$$
\ln w=\alpha E^{*}+\gamma Z+\delta \lambda+\varepsilon
$$

\section{RESULTADOS}

\section{Tipo de emparejamiento}

El cuadro 1 resume los resultados encontrados para los tipos de emparejamiento según las diferentes metodologías. Como era previsible, el método estadístico genera menores tasas de sub y sobrecalificación y se observan diferencias significativas entre los resultados. Además, los rangos estimados son consistentes con la literatura (Alpin, Shackleton, \& Walsh, 1998; Quintini, 2011), excepto para la sobrecalificación mediante el método normativo. Esta diferencia obedece a que la mayoría de la literatura estudia países desarrollados en donde la sobrecalificación ronda el 23\%, mientras que en este caso se estudia un país subdesarrollado caracterizado por mano de obra con bajos niveles de competencias. Así, el porcentaje de subcalificación de 28,9\% estimado mediante el método normativo se encuentra en el rango superior observado en la literatura y muy por encima del promedio, mientras que la tasa de sobrecalificación de 14,2\% refleja, precisamente, esa falta de inversión en capital humano. Por su parte, la tasa de sobreeducación (25,6\%) estimada mediante el método estadístico es similar a la tasa de sobrecalificación (24,1\%). Además, al descomponer la sobrecalificación, se observa que esta corresponde a una aparente sobrecalificación prácticamente en su totalidad, debido a que la mayoría de personas reporta satisfacción con su trabajo, lo cual sugiere que este grupo sí está ubicado en un puesto coherente con sus habilidades. Lo anterior es consistente con una mano de obra con menores habilidades, producto de un sistema educativo deficiente. El método normativo, además, ofrece una ventaja importante respecto a las estimaciones estadísticas: al utilizar parámetros internacionales sobre el nivel de competencia asociado a las ocupaciones, esto permite comparar la situación relativa del mercado nacional con respecto a esos requisitos internacionales; lo cual posibilita determinar la excesiva subcalificación en el mercado costarricense, señalando así la necesidad de mejorar los mecanismos de capacitación de la mano de obra. Por tanto, de aquí en adelante se presentarán los resultados con base en el método normativo, debido a que su construcción es la más objetiva.

6 Adicionalmente, se realizaron pruebas con el ajuste por autoselección de la muestra por la probabilidad de trabajar, pero los coeficientes asociados no fueron significativos, por lo que las ecuaciones salariales se reportan sin este ajuste. 
CUADRO 1

DISTRIBUCIÓN DE LA POBLACIÓN OCUPADA, POR TIPO DE EMPAREJAMIENTO LABORAL

\begin{tabular}{lrrr}
\hline \multicolumn{1}{c}{ Variable } & $\begin{array}{r}\text { Normativo } \\
\text { - ISCO-08 }\end{array}$ & $\begin{array}{r}\text { Estadístico } \\
\text { - ISCO-08 }\end{array}$ & $\begin{array}{c}\text { Estadístico - Años } \\
\text { de educación }\end{array}$ \\
\hline Subcalificación & 28,9 & 7,9 & 14,7 \\
Calificación & 56,9 & 68,1 & 59,7 \\
Sobrecalificación & 14,2 & 24,1 & 25,6 \\
Aparentemente sobrecalificación & 13,3 & 23,0 & 24,4 \\
Genuina sobrecalificación & 0,8 & 1,0 & 1,2 \\
\hline
\end{tabular}

Fuente: Elaboración propia con datos de ENAHO.

El gráfico 1 presenta la evolución del tipo de emparejamiento. En general, las tasas de los diversos tipos de emparejamiento son bastante estables para el período, aunque se observa una leve disminución de la tasa de subcalificación entre 2011 y 2014, así como un leve aumento en la tasa de aparente sobrecalificación a partir de 2015 y que se estabiliza rápidamente en alrededor de 14\%. Estas variaciones, sin embargo, no constituyen tendencias $y$, por el contrario, pareciera que esta distribución refleja una cierta estabilidad en cuanto al funcionamiento del mercado.

\section{GRÁFICO 1 \\ TIPO DE EMPAREJAMIENTO LABORAL EN LA POBLACIÓN MAYOR DE EDAD Y OCUPADA.} PERÍODO 2011-2017.

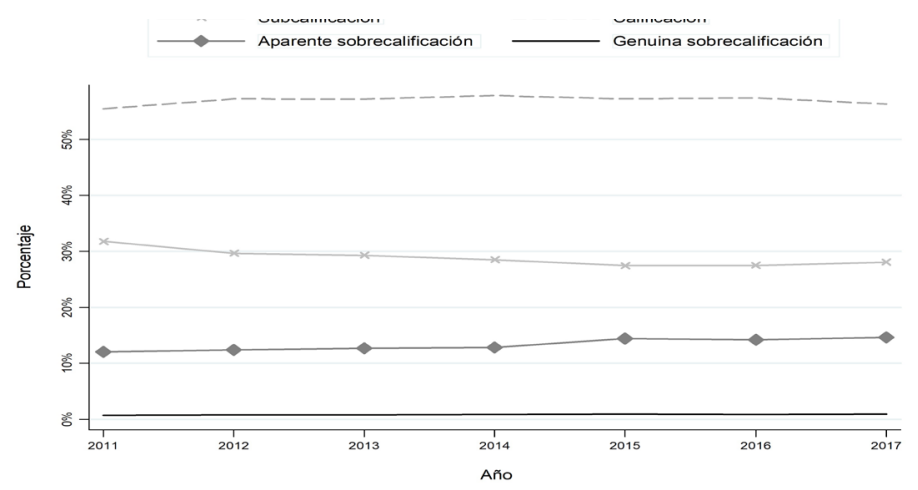

Fuente: Elaboración propia con datos de ENAHO.

El gráfico 2 presenta la distribución de los tipos de emparejamiento a lo largo de las diferentes ocupaciones. Las mayores tasas de una adecuada calificación se observan en los puestos profesionales (92\%) y gerenciales (77,9\%). Estos mismos puestos, junto con el de apoyo administrativo, también presentan las menores tasas de subcalificación, probablemente, debido a que dichos puestos exijan, en la mayoría de los casos, la respectiva titulación o colegiatura. En contraste, las mayores tasas de subcalificación se encuentran en puestos técnicos y profesionales de nivel intermedio (65,9\%), lo cual resultaba previsible, dado el bajo porcentaje de personas con estudios técnicos o parauniversitarios (i.e., con un nivel de competencias 3). Esto se agrava al considerar que $28,6 \%$ de quienes laboran en estos puestos se encuentran en aparente 0 genuina sobrecalificación y solo el 5,5\% adquirió la calificación correspondiente. Evidentemente, esto señala un amplio vacío en la calidad de la oferta laboral, particularmente la técnica, con sus respectivos costos: estos trabajos deben ser asumidos por personas menos calificadas que requerirán capacitación y no han desarrollado las habilidades para realizar el trabajo o debe recurrirse a personal que habrá sobre invertido para desarrollar esa habilidad. 


\section{GRÁFICO 2}

TIPO DE EMPAREJAMIENTO LABORAL, SEGÚN OCUPACIÓN. PERÍODO 2011-2017.

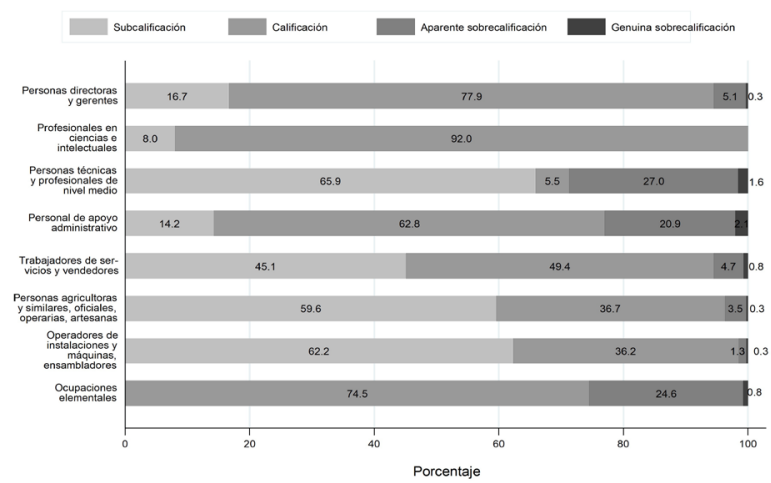

Fuente: Elaboración propia con datos de ENAHO.

También se observan altas tasas de subcalificación en ocupaciones que requieren un menor nivel de competencias, tales como instaladores, operadores de máquinas y ensambladores $(62,2 \%)$, trabajos agrícolas $(59,6 \%)$ y trabajadores de servicios y vendedores $(45,1 \%)$, evidenciando la necesidad de ofrecer educación superior técnica. Como ya se mencionó, la sobrecalificación se concentra en puestos técnicos de nivel intermedio (27\%) y en puestos de apoyo administrativo (20,9\%). En estas ocupaciones, al menos $20 \%$ de los puestos son tomados por personas con un nivel de competencia 3 o 4, que, sin embargo, se encuentran satisfechas con su trabajo, señalizando un menor nivel de habilidad. Por su parte, las ocupaciones elementales también reportan una alta tasa de aparente sobrecalificación $(24,6 \%)$, la cual es llenada por personas con un nivel de competencias 2. Esto es consistente con la literatura: dado que los estudios secundarios suponen una etapa de transición, las personas que no logran concluir esa transición hacia estudios terciarios son más vulnerables debido a su falta de especialización (Montenegro \& Patrinos, 2013), lo que, en este caso, se observa en una probabilidad de alrededor de $18 \%$ de desempeñar tanto puestos elementales (nivel de competencia 1), como puestos técnicos (nivel 3).

\section{GRÁFICO 3}

SALARIO PROMEDIO REAL POR HORA DE LA POBLACIÓN OCUPADA, SEGÚN NIVEL DE COMPETENCIA Y TIPO DE EMPAREJAMIENTO. PERÍODO 2011-2017.

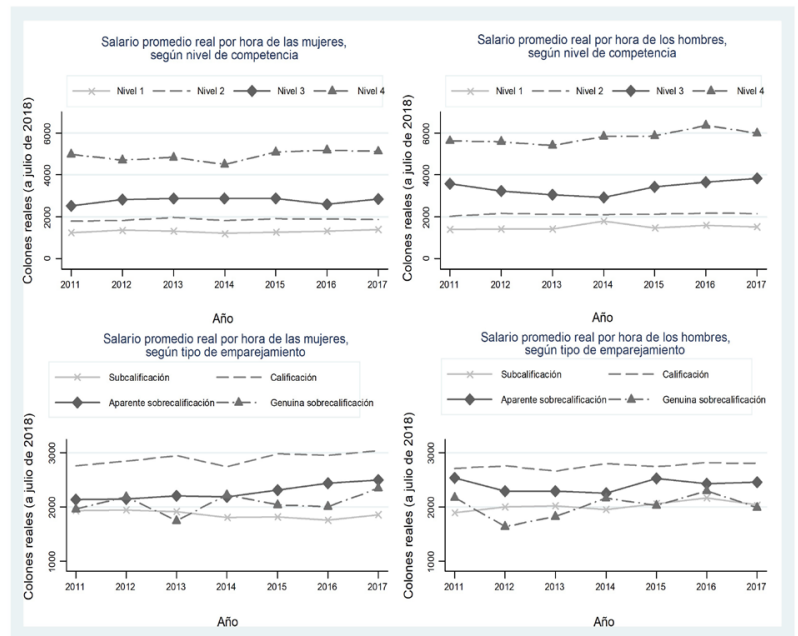

Fuente: Elaboración propia con datos de ENAHO. 
Lo anterior evidentemente repercute sobre las condiciones salariales: los salarios reales promedios por hora que se observan son crecientes con respecto al nivel de competencia, tanto para mujeres como hombres (gráfico 3) y, en ambos casos, se observa una cierta estabilidad del salario a lo largo del tiempo, así como un nivel salarial superior para los hombres en todos los casos. Nótese, además, que no solo los salarios son crecientes, sino que las brechas entre los niveles de competencia también lo son, i.e., existen rendimientos crecientes respecto al nivel de competencia, los cuales se explicarían, al menos en parte, por el fuerte y rápido desgranado del sistema educativo. Ahora bien, las mujeres cuyo nivel de competencias coincide con el requerido para su puesto de trabajo son quienes reportan el mayor nivel de salario. Asimismo, tanto este grupo como el de mujeres con algún grado de sobrecalificación registran una tasa de crecimiento salarial. Por el contrario, los salarios de los hombres según su tipo de emparejamiento son estables, con excepción del salario para los hombres subcalificados que sí registra un crecimiento promedio positivo, aunque reducido (de apenas 1,3\% anual). Las personas aparentemente sobrecalificadas reportan un salario por hora inferior al de las personas calificadas, pero superior al de aquellas que se encuentran genuinamente sobrecalificadas, lo cual es consistente con la literatura. En la muestra femenina, las mujeres subcalificadas son quienes enfrentan el menor salario promedio por hora, tal y como se hubiese esperado; pero este no es el caso para los hombres subcalificados, quienes ganan en forma similar a los hombres aparentemente sobrecalificados. COMPETENCIA Y TIPO DE EMPAREJAMIENTO. PERÍODO 2011-2017.

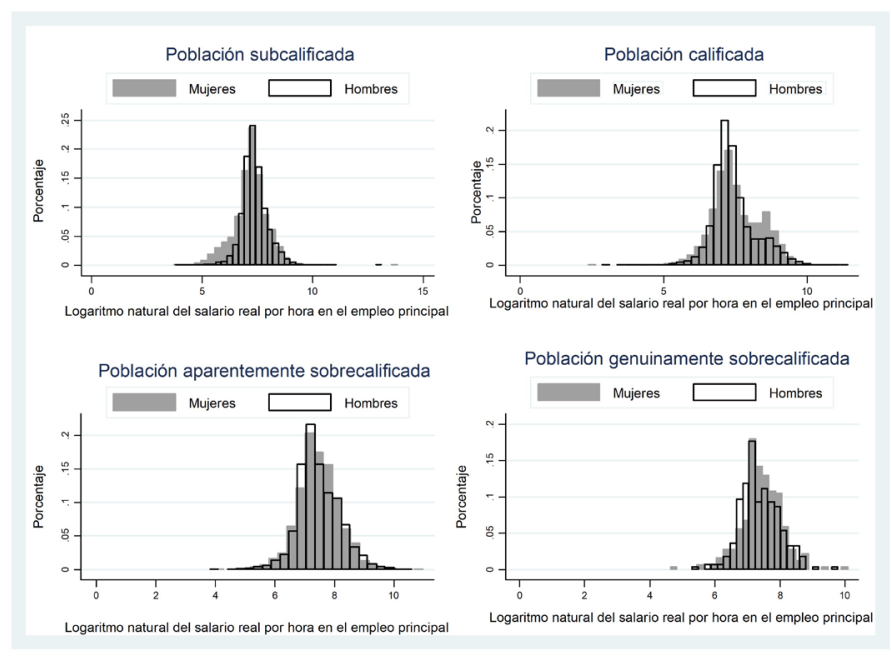

Fuente: Elaboración propia con datos de ENAHO.

El gráfico 4 muestra la distribución porcentual del logaritmo de los salarios reales por hora según género y tipo de emparejamiento. Para la población subcalificada, que aglutina a una quinta parte de las mujeres ocupadas y 35\% de los hombres, la distribución salarial masculina presenta una mayor curtosis (6) que la distribución femenina $(4,9)$. Además, se observa un mayor porcentaje de mujeres subcalificadas con salarios bajos que los hombres, lo cual se refleja en un salario medio inferior para ellas. Para la población calificada, la distribución femenina se ubica más hacia la derecha y presenta una menor curtosis $(3,2)$ que la de los hombres $(4,3)$, así como una menor asimetría $(0,15$ vs. 0,67$)$. En consecuencia, el salario medio por hora de las mujeres (2903 CRC reales) supera al de los hombres (2759 CRC reales) y su peso es tal que el salario 
promedio observado para las mujeres en su totalidad supera al de todos los hombres, ya que es el grupo de mayor tamaño y concentra al $61,9 \%$ de las mujeres y al 53,4\% de los hombres. Por su parte, la distribución de la población con aparente sobrecalificación es similar entre mujeres y hombres, mientras que en el grupo de genuina sobrecalificación la distribución femenina presenta una asimetría negativa y mayor curtosis $(5,0)$ que los hombres $(3,8)$, así como un mayor salario promedio (2210 CRC reales) que el de los hombres (2078 CRC reales). Así, tanto para la población adecuadamente calificada como para la genuinamente sobrecalificada, las mujeres reportan un salario medio superior que los hombres.

\section{Logit multinomial para el tipo de emparejamiento: probabilidad de subcalificación}

El cuadro 2 reporta los efectos marginales medios de la ecuación logit multinomial definida en (ii) para la población ocupada 7 . Conforme a la literatura, la decisión de ocupación se asocia con el tipo de emparejamiento laboral (Alpin, Shackleton, \& Walsh, 1998; Battu, Belfield, \& Sloane, 1999). Trabajar en labores agrícolas, ser oficial, o persona operaria o artesana de artes mecánicas $y$ de otros oficios, u operadora de instalaciones y máquinas, ser ensamblador o personal técnico o profesional de nivel medio incrementa la probabilidad de que la persona esté subcalificada para su puesto de trabajo relativo a quienes trabajan en servicios, comercio y mercados. Como se recordará, estas son las ocupaciones con mayor nivel de subcalificación debido a la escasez de mano de obra con niveles de competencia 3 y, en algún grado, 2. Ocupar una dirección o gerencia o ser profesional se relaciona negativamente con la probabilidad de subcalificación para la muestra agregada y los hombres, relativo al grupo de referencia. Por el contrario, el signo positivo para la muestra femenina es contraintuitivo, en particular si se considera que $93 \%$ las mujeres en estos puestos cuentan con un nivel de competencia 4. En realidad, lo que ocurre es que esta variable cambia de signo (de negativo a positivo) cuando se introduce la variable que identifica a las jefas de hogar. Una posible explicación es que las mujeres con ciertas condiciones de acceso al mercado laboral pueden ocupar estos puestos una vez que se vean obligadas a asumir la jefatura de su hogar. Por ejemplo, podrían incorporarse a una empresa familiar o verse presionadas a abrir su propia empresa y asumir, por tanto, su gerencia.

Contar con un trabajo permanente disminuye la probabilidad de subcalificación para la muestra total y la masculina, aportando evidencia a favor del argumento de que las personas sean más proclives a aceptar un mal emparejamiento cuando este es transitorio. Además, la probabilidad de subcalificación se reduce en forma creciente conforme aumenta el tamaño de la empresa, lo que apoya la hipótesis de que los empleos más informales y precarios se asocian con las microempresas. Por el contrario, trabajar en el sector privado o en un hogar privado y ser cabeza del hogar se relacionan positivamente con la subcalificación.

Tener pareja reduce la probabilidad de subcalificación en 17 p.p. para las mujeres y contar con una persona en edad preescolar adicional en el hogar lo hace en 8,3 p.p., probablemente porque, en el primer caso, esto les permite autoseleccionarse para entrar al mercado laboral y les facilita escoger un trabajo más acorde con su nivel de competencias y, en el segundo, las inclina a retirarse del mercado laboral si sus opciones de trabajo no concuerdan con sus competencias. En contraste, tener pareja (1 p.p.) y contar con 1 preescolar adicional en el hogar (1,5 p.p.) incrementan levemente la probabilidad de los hombres de estar subcalificados. Nuevamente, esto podría asociarse a una mayor presión para ser el proveedor del hogar. La edad se relaciona positiva y decrecientemente con la probabilidad de las mujeres de estar subcalificadas, pero no es significativa para los hombres

$7 \quad$ Por cuestión de espacio, se discute únicamente el modelo agregado, pero las estimaciones se realizaron para cada año y están disponibles a solicitud. De estas se obtuvo la inversa de la razón de Mills para el ajuste por endogeneidad que se utilizó en la estimación de ingresos. 
al nivel de confianza estándar. Al respecto, es posible que las mujeres con mayor arraigo en el mercado laboral sean más hábiles y disfruten de mejores oportunidades de ascenso, lo que se plasma en una mayor probabilidad de subcalificación.

En general, ser mujer reduce en 16 p.p. la probabilidad de subcalificación y el tamaño del hogar la aumenta (1 p.p.), probablemente porque genera mayor presión para aceptar un puesto de trabajo y contribuir en el hogar. Contar con otras personas desempleadas o inactivas en el hogar $y$ poseer un trabajo parcial no resultan significativas al nivel de significancia estándar. La región de residencia $y$ vivir en zona urbana también afectan en forma diferente a mujeres $y$ hombres. Igualmente, ser migrante incrementa la probabilidad de subcalificación para las mujeres en 10,1 p.p., mientras que la reduce para los hombres (2,1 p.p.), indicando que, probablemente, las mujeres migrantes enfrenten mayores barreras de entrada o de búsqueda en el mercado laboral que los hombres migrantes. Y, finalmente, la inversa de la razón de Mills es significativa al 1\% en todos los casos, indicando que efectivamente existe un proceso de auto selección al escoger el trabajo y el consecuente tipo de emparejamiento.

\section{Logit multinomial para el tipo de emparejamiento: probabilidad de aparente sobrecalificación}

Como sería esperable y salvo algunas excepciones, los signos asociados a las variables dependientes de la regresión se invierten en la estimación de la probabilidad de aparente sobrecalificación, confirmando el análisis de la sección anterior, en particular aquel relacionado con la persistencia de los roles de género. Así, se encuentra evidencia a favor de la tesis sostenida por Jauhiainen (2011), según la cual las mujeres priorizarían el trabajo de sus maridos y limitarían su espectro de búsqueda de trabajo al de él, pues las mujeres con pareja muestran una probabilidad superior en 22,4 p.p. a estar aparentemente sobrecalificadas en comparación con las solteras. Las mujeres con una persona adicional menor de 6 años en el hogar también ven incrementada su probabilidad de aparente sobrecalificación en 8,1 p.p., sugiriendo que ellas ajustan sus decisiones laborales y aceptan trabajos con menores requerimientos, posiblemente para priorizar el cuido de menores.

En concordancia, la probabilidad de aparente sobrecalificación se relaciona negativamente con el tamaño del hogar. Esto podría reflejar el hecho de que las mujeres menos educadas tienen hogares más numerosos y, por tanto, una menor probabilidad de estar sobrecalificadas, pero también podría sugerir que, al incrementarse el tamaño del hogar la presión por el cuido aumenta y desalienta a las mujeres de participar en el mercado laboral y, al retirarse, se observaría una relación negativa entre estas variables. Lo anterior también explicaría la relación negativa y creciente entre la edad y la probabilidad de una aparente sobrecalificación, pues para reincorporarse al mercado laboral, las mujeres estarían exigiendo hacerlo en puestos adecuados a sus competencias, no puestos inferiores. Este hecho se diferencia de lo observado en los países europeos (ILO, 2014; Jauhiainen, 2011), pues al tener intermitencia laboral lo usual es que las mujeres se reincorporen en trabajos inferiores. Sin embargo, esto no estaría ocurriendo en el caso costarricense por el alto precio de reserva de las mujeres.

Ahora bien, el hecho de que ser mujer incrementa la probabilidad de aparente sobrecalificación en 19,8 p.p. también podría reflejar un componente discriminatorio contra las mujeres, i.e., podría estárseles exigiendo una mayor preparación para los puestos de trabajo que a los hombres. Las mujeres migrantes, en contraste, tienen una menor probabilidad de estar aparentemente sobrecalificadas que las nacionales, mientras que, para los hombres migrantes, la relación es positiva. 


\section{CUADRO 2}

EFECTOS MARGINALES MEDIOS PARA LA LOGIT MULTINOMIAL SOBRE EL TIPO DE EMPAREJAMIENTO LABORAL DE LA POBLACIÓN MAYOR DE EDAD OCUPADA, POR GÉNERO (ERRORES ESTÁNDAR ROBUSTOS) PERÍODO: 2011-2017.

\begin{tabular}{|c|c|c|c|c|c|c|c|c|c|}
\hline & \multicolumn{3}{|c|}{ TOTAL } & \multicolumn{3}{|c|}{ MUJERES } & \multicolumn{3}{|c|}{ HOMBRES } \\
\hline & $\begin{array}{l}\text { Sub- } \\
\text { calificación }\end{array}$ & $\begin{array}{c}\text { Aparente } \\
\text { sobre- } \\
\text { calificación }\end{array}$ & $\begin{array}{c}\text { Sobre } \\
\text { calificación }\end{array}$ & $\begin{array}{l}\text { Sub- } \\
\text { calificación }\end{array}$ & $\begin{array}{c}\text { Aparente } \\
\text { sobre- } \\
\text { calificación }\end{array}$ & $\begin{array}{c}\text { Sobre } \\
\text { calificación }\end{array}$ & $\begin{array}{l}\text { Sub- } \\
\text { calificación }\end{array}$ & $\begin{array}{c}\text { Aparente } \\
\text { sobre- } \\
\text { calificación }\end{array}$ & $\begin{array}{c}\text { Sobre } \\
\text { calificación }\end{array}$ \\
\hline \multicolumn{10}{|l|}{$\begin{array}{l}\text { Ocupación (base }= \\
\text { servicios, comercios) }\end{array}$} \\
\hline \multirow{2}{*}{$\begin{array}{l}\text { Personas directoras } \\
y \text { gerentes }\end{array}$} & $-0,201 * * *$ & $-0,038^{* * *}$ & $-0,007 * * *$ & $0,331 * * *$ & $-0,223^{* * *}$ & $-0,043^{* * *}$ & $-0,294 * * *$ & 0,009 & 0,001 \\
\hline & $(0,012)$ & $(0,004)$ & $(0,002)$ & $(0,008)$ & $(0,006)$ & $(0,006)$ & $(0,015)$ & $(0,007)$ & $(0,003)$ \\
\hline \multirow{2}{*}{$\begin{array}{l}\text { Profesionales } \\
\text { en ciencias e } \\
\text { intelectuales }\end{array}$} & $-0,262 * * *$ & $-0,063^{* * *}$ & $-0,010 * * *$ & $0,293 * * *$ & $-0,232 * * *$ & $-0,043^{* * *}$ & $-0,333 * * *$ & $-0,037^{* * *}$ & $-0,005^{* * *}$ \\
\hline & $(0,007)$ & $(0,002)$ & $(0,001)$ & $(0,007)$ & $(0,006)$ & $(0,006)$ & $(0,009)$ & $(0,002)$ & $(0,001)$ \\
\hline \multirow{2}{*}{$\begin{array}{l}\text { Personas técnicas } \\
\text { y profesionales de } \\
\text { nivel medio }\end{array}$} & $0,292 * * *$ & $0,140 * * *$ & 0,002 & $0,332^{* * *}$ & $0,016^{* *}$ & $-0,031 * * *$ & $0,300 * * *$ & $0,121^{* * *}$ & $0,005^{* * *}$ \\
\hline & $(0,006)$ & $(0,004)$ & $(0,002)$ & $(0,004)$ & $(0,006)$ & $(0,006)$ & $(0,008)$ & $(0,005)$ & $(0,002)$ \\
\hline \multirow{2}{*}{$\begin{array}{l}\text { Personal de apoyo } \\
\text { administrativo }\end{array}$} & $-0,214 * * *$ & $0,105^{* * *}$ & $0,003 * *$ & $-0,138 * * *$ & $0,035^{* * *}$ & $-0,021 * * *$ & $-0,187 * * *$ & 0,078 **** & 0,002 \\
\hline & $(0,007)$ & $(0,004)$ & $(0,002)$ & $(0,007)$ & $(0,006)$ & $(0,006)$ & $(0,010)$ & $(0,005)$ & $(0,001)$ \\
\hline \multirow{2}{*}{$\begin{array}{l}\text { Personas } \\
\text { agricultoras y } \\
\text { similares, oficiales, } \\
\text { operarias y } \\
\text { artesanas de artes } \\
\text { mecánicas y de otros } \\
\text { oficios }\end{array}$} & $0,131 * * *$ & $-0,004$ & $-0,006^{* * *}$ & $0,047 * * *$ & $0,023^{*}$ & $-0,021 *$ & $0,122 * * *$ & $-0,007^{* *}$ & $-0,003^{* * *}$ \\
\hline & $(0,007)$ & 0,004 & 0,001 & 0,011 & 0,013 & 0,012 & 0,008 & 0,003 & 0,001 \\
\hline \multirow{2}{*}{$\begin{array}{l}\text { Operadores de } \\
\text { instalaciones } \\
\text { y máquinas y } \\
\text { ensambladores }\end{array}$} & $0,158 * * *$ & $-0,034 * * *$ & $-0,005 * * *$ & $0,057 * * *$ & $-0,010$ & 0,002 & $0,155^{* * *}$ & $-0,023^{* * *}$ & $-0,003^{* *}$ \\
\hline & $(0,008)$ & $(0,004)$ & $(0,002)$ & $(0,011)$ & $(0,024)$ & $(0,022)$ & $(0,009)$ & $(0,003)$ & $(0,001)$ \\
\hline \multirow{2}{*}{$\begin{array}{l}\text { Ocupaciones } \\
\text { elementales }\end{array}$} & $-0,446 * * *$ & $0,235^{* * *}$ & 0,001 & $-0,368 * * *$ & $0,301 * * *$ & $-0,016^{* *}$ & $-0,493 * * *$ & $0,193^{* * *}$ & $0,004^{* * *}$ \\
\hline & $(0,004)$ & $(0,004)$ & $(0,001)$ & $(0,003)$ & $(0,008)$ & $(0,007)$ & $(0,006)$ & $(0,005)$ & $(0,001)$ \\
\hline \multirow[t]{2}{*}{ Trabajo permanente } & $-0,053 * * *$ & $0,026 * * *$ & $-0,002$ & $-0,011$ & $0,011^{*}$ & $-0,006^{*}$ & $-0,064 * * *$ & $0,023^{* * *} *$ & 0,000 \\
\hline & $(0,007)$ & $(0,004)$ & $(0,001)$ & $(0,007)$ & $(0,006)$ & $(0,003)$ & $(0,009)$ & $(0,004)$ & $(0,001)$ \\
\hline \multirow[t]{2}{*}{ Trabajo parcial } & 0,003 & $0,013 * * *$ & 0,002 & 0,005 & 0,001 & 0,000 & 0,010 & 0,003 & 0,002 \\
\hline & $(0,004)$ & $(0,003)$ & $(0,001)$ & $(0,003)$ & $(0,003)$ & $(0,002)$ & $(0,007)$ & $(0,004)$ & $(0,001)$ \\
\hline \multicolumn{10}{|c|}{$\begin{array}{l}\text { Tamaño de la empresa } \\
\text { (base= microempresa) }\end{array}$} \\
\hline \multirow[t]{2}{*}{ Pequeña } & $-0,015^{* * *}$ & $0,021 * * *$ & 0,000 & $-0,011 * * *$ & $-0,001$ & $-0,000$ & $-0,020 * * *$ & $0,023^{* * *} *$ & $-0,000$ \\
\hline & $(0,004)$ & $(0,003)$ & $(0,001)$ & $(0,004)$ & $(0,004)$ & $(0,002)$ & $(0,005)$ & $(0,004)$ & $(0,001)$ \\
\hline \multirow[t]{2}{*}{ Mediana } & $-0,022^{* * *}$ & $0,026 * * *$ & 0,000 & $-0,012 * * *$ & 0,000 & 0,001 & $-0,027 * * *$ & $0,025^{* * * *}$ & $-0,001$ \\
\hline & $(0,004)$ & $(0,004)$ & $(0,001)$ & $(0,005)$ & $(0,005)$ & $(0,002)$ & $(0,005)$ & $(0,004)$ & $(0,001)$ \\
\hline \multirow[t]{2}{*}{ Grande } & $-0,046^{* * *}$ & $0,026 * * *$ & $0,002 *$ & $-0,029 * * *$ & 0,002 & 0,001 & $-0,050 * * *$ & 0,029 *** & 0,002 \\
\hline & $(0,004)$ & $(0,003)$ & $(0,001)$ & $(0,004)$ & $(0,004)$ & $(0,002)$ & $(0,005)$ & $(0,004)$ & $(0,001)$ \\
\hline \multirow[t]{2}{*}{$\begin{array}{l}\text { Trabaja en el sector } \\
\text { privado }\end{array}$} & $0,098 * * *$ & $-0,062^{* * * *}$ & $-0,002 *$ & $0,033 * * *$ & $-0,013 * * * *$ & $-0,001$ & $0,122^{* * * *}$ & $-0,062 * * *$ & 0,001 \\
\hline & $(0,003)$ & $(0,004)$ & $(0,001)$ & $(0,003)$ & $(0,004)$ & $(0,002)$ & $(0,005)$ & $(0,005)$ & $(0,001)$ \\
\hline \multirow[t]{2}{*}{$\begin{array}{l}\text { Trabaja en hogar } \\
\text { privado }\end{array}$} & $0,046^{* * * *}$ & $0,014 * * *$ & 0,003 & 0,016 **** & $-0,009 * *$ & 0,003 & $0,045^{*}$ & $-0,011$ & $-0,000$ \\
\hline & $(0,007)$ & $(0,005)$ & $(0,002)$ & $(0,005)$ & $(0,004)$ & $(0,003)$ & $(0,026)$ & $(0,011)$ & $(0,003)$ \\
\hline \multirow[t]{2}{*}{ Es cabeza del hogar } & $0,116^{* * * *}$ & $-0,129 * * * *$ & $-0,010 * * *$ & $0,302 * * *$ & $-0,267 * * *$ & $-0,020$ & $0,013 * *$ & $-0,019 * * *$ & $-0,001$ \\
\hline & $(0,004)$ & $(0,003)$ & $(0,001)$ & $(0,003)$ & $(0,003)$ & $(0,002)$ & $(0,005)$ & $(0,004)$ & $(0,001)$ \\
\hline
\end{tabular}




\begin{tabular}{|c|c|c|c|c|c|c|c|c|c|}
\hline & \multicolumn{3}{|c|}{ TOTAL } & \multicolumn{3}{|c|}{ MUJERES } & \multicolumn{3}{|c|}{ HOMBRES } \\
\hline & $\begin{array}{l}\text { Sub- } \\
\text { calificación }\end{array}$ & $\begin{array}{l}\text { Aparente } \\
\text { sobre- } \\
\text { calificación }\end{array}$ & $\begin{array}{c}\text { Sobre } \\
\text { calificación }\end{array}$ & $\begin{array}{l}\text { Sub- } \\
\text { calificación }\end{array}$ & $\begin{array}{l}\text { Aparente } \\
\text { sobre- } \\
\text { calificación }\end{array}$ & $\begin{array}{c}\text { Sobre } \\
\text { calificación }\end{array}$ & $\begin{array}{l}\text { Sub- } \\
\text { calificación }\end{array}$ & $\begin{array}{l}\text { Aparente } \\
\text { sobre- } \\
\text { calificación }\end{array}$ & $\begin{array}{c}\text { Sobre } \\
\text { calificación }\end{array}$ \\
\hline \multirow[t]{2}{*}{ Tiene pareja } & $0,008^{* * *}$ & $-0,000$ & $-0,002 * * *$ & $-0,170 * * *$ & $0,224 * * *$ & $0,020 * * *$ & $0,010^{*}$ & $-0,011 * * *$ & $-0,001$ \\
\hline & $(0,003)$ & $(0,002)$ & $(0,001)$ & $(0,003)$ & $(0,005)$ & $(0,003)$ & $(0,005)$ & $(0,004)$ & $(0,001)$ \\
\hline \multirow[t]{2}{*}{ Es migrante } & 0,002 & $-0,015^{* * *}$ & $-0,005^{* * *}$ & $0,101^{* * *}$ & $-0,084 * * *$ & $-0,010 * * *$ & $-0,021 * * *$ & 0,006 & $-0,003 * * *$ \\
\hline & $(0,005)$ & $(0,003)$ & $(0,001)$ & $(0,006)$ & $(0,003)$ & $(0,001)$ & $(0,006)$ & $(0,004)$ & $(0,001)$ \\
\hline \multirow[t]{2}{*}{ Es mujer } & $-0,160 * * *$ & $0,198 * * *$ & $0,017^{* * *}$ & & & & & & \\
\hline & $(0,003)$ & $(0,005)$ & $(0,002)$ & & & & & & \\
\hline \multirow{2}{*}{$\begin{array}{l}\text { Cantidad de } \\
\text { menores de } 6 \text { años } \\
\text { en el hogar }\end{array}$} & $0,008^{* * *}$ & $-0,004^{*}$ & $-0,002^{* * *}$ & $-0,083^{* * *}$ & $0,081^{* * *}$ & 0,002 & $0,015 * * *$ & $-0,006^{* *}$ & $-0,001$ \\
\hline & $(0,002)$ & $(0,002)$ & $(0,001)$ & $(0,003)$ & $(0,003)$ & $(0,001)$ & $(0,003)$ & $(0,003)$ & $(0,001)$ \\
\hline \multirow[t]{2}{*}{ Tamaño del hogar } & $0,010^{* * *}$ & $-0,006^{* * *}$ & $-0,001^{*}$ & $0,008 * * *$ & $-0,005^{* * * *}$ & $-0,001 *$ & $0,008 * * *$ & $-0,004 * * *$ & $-0,000$ \\
\hline & $(0,001)$ & $(0,001)$ & $(0,000)$ & $(0,001)$ & $(0,001)$ & $(0,001)$ & $(0,001)$ & $(0,001)$ & $(0,000)$ \\
\hline \multirow{2}{*}{$\begin{array}{l}\text { Personas } \\
\text { desempleadas o } \\
\text { inactivas en el hogar }\end{array}$} & $0,003^{*}$ & $-0,008^{* * *}$ & $-0,001^{* *}$ & $-0,003^{*}$ & 0,001 & $-0,000$ & 0,001 & $-0,004 * *$ & $-0,001^{*}$ \\
\hline & $(0,002)$ & $(0,001)$ & $(0,000)$ & $(0,002)$ & $(0,002)$ & $(0,001)$ & $(0,002)$ & $(0,002)$ & $(0,000)$ \\
\hline \multirow[t]{2}{*}{ Edad } & $0,023 * * *$ & $-0,021^{* * *}$ & $-0,001 * * *$ & $0,076^{* * *}$ & $-0,066^{* * *}$ & $-0,003 * * *$ & $-0,002^{*}$ & 0,000 & $0,001 * * *$ \\
\hline & $(0,001)$ & $(0,001)$ & $(0,000)$ & $(0,001)$ & $(0,001)$ & $(0,001)$ & $(0,001)$ & $(0,001)$ & $(0,000)$ \\
\hline \multirow[t]{2}{*}{ Edad al cuadrado } & $-0,000^{* * *}$ & $0,000 * * *$ & 0,000 & $-0,001 * * *$ & $0,001 * * *$ & $0,000 * * *$ & $0,000 * * *$ & $-0,000^{*}$ & $-0,000 * * *$ \\
\hline & $(0,000)$ & $(0,000)$ & $(0,000)$ & $(0,000)$ & $(0,000)$ & $(0,000)$ & $(0,000)$ & $(0,000)$ & $(0,000)$ \\
\hline \multicolumn{10}{|l|}{$\begin{array}{l}\text { Región (referencia }= \\
\text { Central) }\end{array}$} \\
\hline \multirow[t]{2}{*}{ Chorotega } & $-0,044 * * *$ & $0,059 * * *$ & 0,001 & $-0,109 * * *$ & $0,112 * * *$ & 0,002 & $-0,005$ & $0,010^{*}$ & $-0,002$ \\
\hline & $(0,005)$ & $(0,005)$ & $(0,001)$ & $(0,004)$ & $(0,006)$ & $(0,002)$ & $(0,006)$ & $(0,005)$ & $(0,001)$ \\
\hline \multirow[t]{2}{*}{ Pacífico Central } & $-0,004$ & $0,019 * * *$ & 0,001 & $-0,073^{* * *}$ & $0,072 * * *$ & 0,004 & $0,023^{* * *}$ & $-0,014 * * *$ & $-0,002 *$ \\
\hline & $(0,005)$ & $(0,004)$ & $(0,001)$ & $(0,004)$ & $(0,005)$ & $(0,003)$ & $(0,006)$ & $(0,005)$ & $(0,001)$ \\
\hline \multirow[t]{2}{*}{ Brunca } & $-0,046^{* * *}$ & $0,046^{* * *}$ & $0,005^{* * *}$ & $-0,176^{* * *}$ & $0,238 * * *$ & $0,024 * * *$ & $-0,009$ & 0,008 & $-0,001$ \\
\hline & $(0,005)$ & $(0,005)$ & $(0,002)$ & $(0,004)$ & $(0,010)$ & $(0,006)$ & $(0,007)$ & $(0,005)$ & $(0,001)$ \\
\hline \multirow[t]{2}{*}{ Huetar Atlántica } & 0,007 & 0,000 & $-0,001$ & $-0,133^{* * *}$ & $0,172 * * *$ & $0,007 *$ & $0,021 * * *$ & $-0,022 * * *$ & $-0,002$ \\
\hline & $(0,005)$ & $(0,004)$ & $(0,001)$ & $(0,004)$ & $(0,008)$ & $(0,004)$ & $(0,006)$ & $(0,004)$ & $(0,001)$ \\
\hline \multirow[t]{2}{*}{ Huetar Norte } & 0,004 & 0,000 & 0,000 & $-0,089 * * *$ & $0,087 * * * *$ & 0,006 & $0,021^{* * *}$ & $-0,015^{* * * *}$ & $-0,001$ \\
\hline & $(0,005)$ & $(0,004)$ & $(0,001)$ & $(0,004)$ & $(0,006)$ & $(0,003)$ & $(0,006)$ & $(0,005)$ & $(0,001)$ \\
\hline \multirow[t]{2}{*}{ Zona urbana } & $-0,044 * * *$ & $0,035^{* * * *}$ & 0,000 & $0,091 * * *$ & $-0,104$ *** & $-0,011$ & $-0,059 * * *$ & $0,046^{* * * *}$ & $0,002 *$ \\
\hline & $(0,003)$ & $(0,003)$ & $(0,001)$ & $(0,003)$ & $(0,004)$ & $(0,002)$ & $(0,004)$ & $(0,003)$ & $(0,001)$ \\
\hline \multirow[t]{2}{*}{$\begin{array}{l}\text { Inversa de la razón } \\
\text { de Mills }\end{array}$} & $0,269 * * *$ & $-0,319 * * *$ & $-0,027 * * *$ & $0,940 * * *$ & $-0,909 * * *$ & $-0,062$ & $-0,070 * * *$ & 0,012 & $0,009 * * *$ \\
\hline & $(0,007)$ & $(0,006)$ & $(0,002)$ & $(0,013)$ & $(0,010)$ & $(0,005)$ & $(0,013)$ & $(0,010)$ & $(0,002)$ \\
\hline Observaciones & 75612 & 75612 & 75612 & 29688 & 29688 & 29688 & 45924 & 45924 & 45924 \\
\hline
\end{tabular}

Fuente: Elaboración propia con datos de ENAHO. 
Los hombres, en cambio, ven reducida su probabilidad de aparente sobrecalificación conforme tienen pareja (-1,1 p.p.), asumen la jefatura del hogar (-1,9 p.p.), aumenta la cantidad de menores de 6 años en el hogar (-0,6 p.p.) o de personas desempleadas o inactivas en el hogar (-0,4 p.p.) y el tamaño de este (-0,4 p.p.), todas señalizando su importancia como proveedor familiar. Es decir, la presión que ejerce la necesidad de constituirse en proveedor los empuja a tomar trabajos para los cuales están más bien subcalificados y disminuye su probabilidad de sobrecalificación.

En cuanto a las condiciones laborales, las variables presentan el signo opuesto al encontrado para la probabilidad de subcalificación, con algunas excepciones que se discuten a continuación: los trabajos profesionales presentan el signo esperado en todos los casos, i.e., la relación observada es negativa y significativa, particularmente para las mujeres, para quienes la probabilidad de aparente sobrecalificación se reduce en 23,2 p.p., en promedio, relativo a la ocupación de referencia. Igualmente, dicha probabilidad se asocia negativamente con poseer un puesto gerencial o de dirección en el modelo agregado (3,8 p.p.) y el femenino (22,3 p.p.), pero no es significativo para los hombres. Esta probabilidad se relaciona positivamente con trabajar en puestos técnicos y profesionales de nivel intermedio o en apoyo administrativo, afectando con mayor magnitud a los hombres. Que trabajar en puestos técnicos y profesionales de nivel intermedio se asocie positivamente, tanto con la probabilidad de subcalificación como con la probabilidad de aparente sobrecalificación, muestra que estos puestos están siendo llenados con personas con competencias superiores e inferiores a las requeridas, debido al gran vacío en el sistema educativo de ofertas para desarrollar competencias del nivel 3. Las ocupaciones que requieren un nivel 2 de competencias, se asocian negativamente o no se encuentran significativamente asociadas con la probabilidad de aparente sobrecalificación, al nivel de significancia estándar, mientras que trabajar en las ocupaciones elementales obviamente se asocia positivamente con la probabilidad de aparente sobrecalificación.

Tener un trabajo permanente se relaciona positiva y significativamente con la probabilidad de aparente sobrecalificación para los hombres (2,3 p.p.) y la muestra total (2,6 p.p.) a una significancia del $1 \%$ y para las mujeres (1,1 p.p.), al 10\%. Esto es coherente con la literatura (Boll, Leppin, Rossen \& Wolf, 2016), pues conforme se adquiere estabilidad en el empleo, se cuenta con mayor probabilidad de recibir ascensos o capacitaciones en el trabajo. El trabajo parcial también incrementa la probabilidad de aparente sobrecalificación en 1,3 p.p. para la muestra agrupada, lo cual es consistente con la tesis de que las personas pueden aceptar trabajos temporales o parciales para adquirir experiencia. Trabajar en el sector privado se asocia negativamente con la probabilidad de aparente sobrecalificación, sugiriendo que es más difícil ascender en el sector privado si no se cuenta con los requerimientos exigidos por el puesto, mientras que el sector público sería más laxo en este sentido. Por otra parte, no trabajar en microempresas se relaciona positiva $y$ significativamente con la probabilidad de aparente sobrecalificación en el modelo agrupado y masculino, más no así para las mujeres, para quienes el tamaño de la empresa no es significativo. Finalmente, la inversa de la razón de Mills es significativa para el modelo completo y el de mujeres, mas no así el de hombres. Finalmente, la región y zona de residencia también son significativas.

\section{Logit multinomial para el tipo de emparejamiento: probabilidad de genuina sobrecalificación}

El modelo para la probabilidad de genuina sobrecalificación básicamente reproduce las mismas relaciones entre las variables que el modelo para la aparente sobrecalificación, excepto que la significancia se pierde para algunos de estos regresores debido a que son pocos los casos de genuina sobrecalificación observados. Así, para el modelo agregado, se pierde la significancia de contar con un trabajo permanente, el tamaño de la empresa y trabajar en un hogar privado 
en comparación con el modelo anterior. En la muestra femenina, la mayor diferencia entre este modelo y el anterior se observa en las ocupaciones. Con excepción de las personas que operan instalaciones y máquinas o son ensambladoras, todas las otras ocupaciones se asocian negativamente con la probabilidad de genuina sobrecalificación relativo a trabajar en comercios, servicios o mercados. Finalmente, en el modelo masculino, se pierde significancia en los efectos marginales observados para las regiones Chorotega y Brunca y se invierte el signo asociado a ser hombre migrante, i.e., ser hombre migrante se asocia negativamente $(0,3$ p.p.) con la probabilidad de genuina sobrecalificación.

\section{Ecuación de salarios}

El cuadro 3 presenta los resultados de la regresión para el logaritmo natural de los salarios reales por hora especificada en (iii) ${ }^{8}$. Esta es una regresión diferente a una sin regresores. Los resultados se presentan sin el ajuste de Heckman por la probabilidad de trabajar debido a que este término no resultó significativo. Como era de esperarse, los rendimientos asociados al nivel de competencia son positivos, significativos y crecientes en todos los modelos, pero los hombres reportan coeficientes superiores a los de las mujeres en todos los casos. La potencial experiencia laboral se encuentra positiva y significativamente asociada al logaritmo del salario real y, además, es decreciente, de forma que un año adicional de experiencia incrementa el salario, en promedio, en $1,3 \%$. Y, las personas bilingües ganan $20,3 \%$ más que las no bilingües, por lo que todas las variables asociadas al modelo de capital humano se comportan como lo predice la teoría.

La variable de interés también se comporta según lo esperado: comparado con las personas con su mismo nivel de competencia que se encuentran bien empatadas, las personas subcalificadas reciben un premio de alrededor de 30,5\% y los hombres se benefician más de ello (36,6\%) que las mujeres (24,9\%). Este premio es superior al máximo de 12,5\% reportado en un país desarrollado (Alba-Ramírez, 1993) y refleja la escasez de mano de obra calificada, que es el caso opuesto al de los países desarrollados, donde la preocupación es la sobreeducación. En contraste, las personas aparentemente sobrecalificadas ganan $35,2 \%$ menos que las personas con su mismo nivel de competencia con un trabajo acorde a él y su magnitud es similar entre mujeres y hombres. Este valor es cercano, aunque levemente superior, al reportado por Farooq (2015) para Paquistán y se halla muy por encima de los castigos observados en países desarrollados. Nuevamente, esto señala la necesidad de estudiar los mercados en países subdesarrollados, debido a que estos difieren de los desarrollados. Finalmente, las personas genuinamente sobrecalificadas ganan 47,5\% menos que las personas con su mismo nivel de competencia con un trabajo acorde a él, y este castigo es levemente mayor para los hombres $(47,8 \%)$ que las mujeres $(46,7 \%)$.

$8 \mathrm{Al}$ igual que en los casos anteriores, las estimaciones también se hicieron para cada año separadamente 
CUADRO 3

ECUACIÓN PARA EL LOGARITMO NATURAL DEL SALARIO REAL POR HORA, POR GÉNERO (ERRORES ESTÁNDAR ROBUSTOS) PERÍODO: 2011-2017.

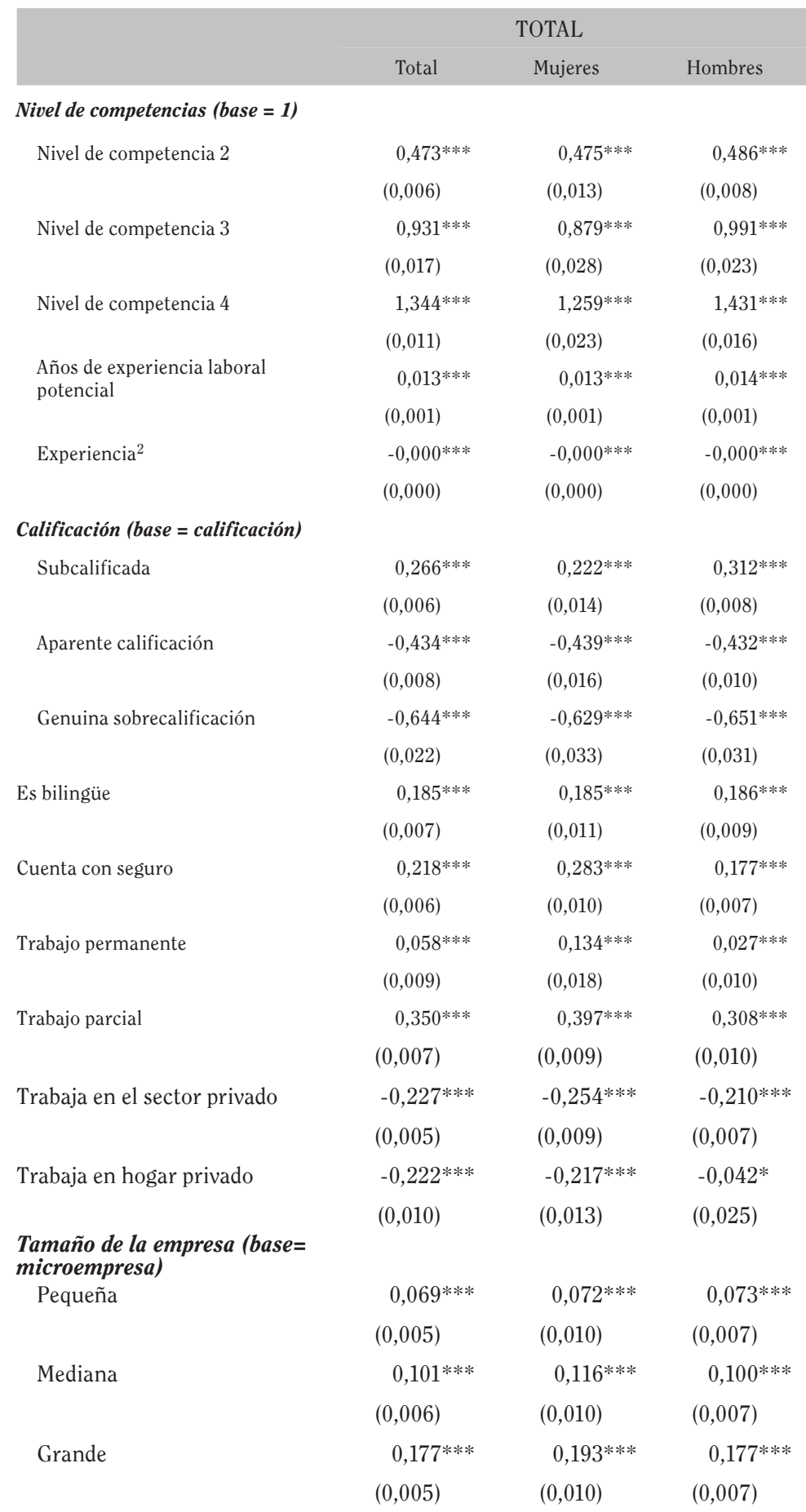




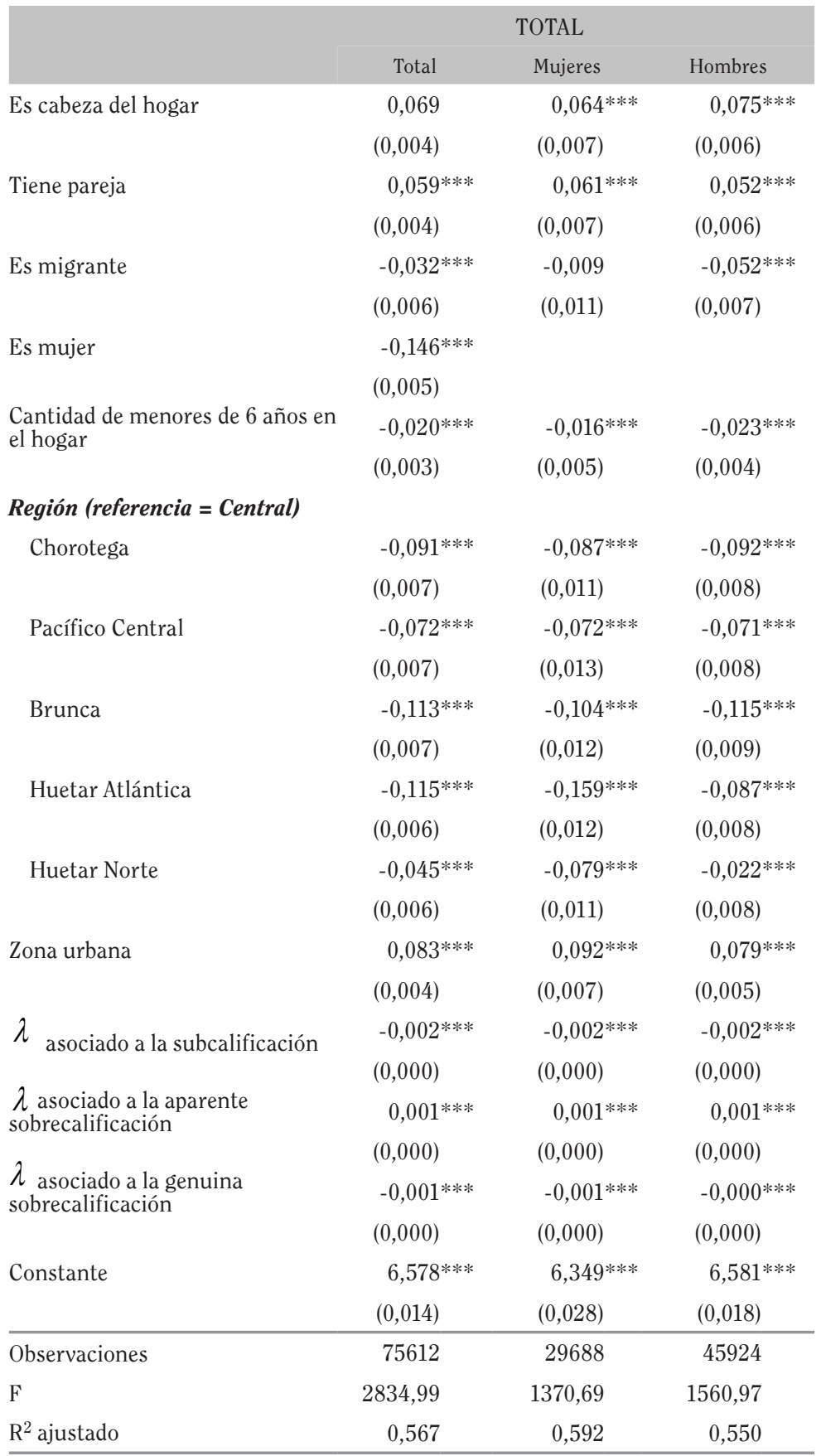

Fuente: Elaboración propia con datos de ENAHO. 
En cuanto a las características relacionadas con el trabajo, contar con seguro en el trabajo se asocia con un incremento de $24,4 \%$ en el salario respecto a quienes no lo tienen. En contraste, trabajar en un hogar privado, usualmente asociado al trabajo doméstico, le reduce el ingreso salarial en $19,5 \%$ a las mujeres y $4,1 \%$ a los hombres en comparación con quienes trabajan en otros lugares. Si estas variables se toman como indicadores de la formalidad en el empleo, las mujeres son quienes más se benefician de un empleo formal. Similarmente, contar con un trabajo permanente se asocia con un incremento salarial promedio de $6 \%$ respecto a quienes cuentan con trabajos temporales $y$, nuevamente, las mujeres reciben un mayor incremento (14,3\%) que los hombres (2,7\%). Esto podría relacionarse con la intermitencia de las mujeres en el mercado laboral que hace que opten por trabajos de menor calidad, de forma que, cuando logran obtener trabajos formales y estables su ingreso se ve incrementado significativamente. Por su parte, el signo positivo del coeficiente asociado a laborar parcialmente es contraintuitivo y difícil de explicar. Podría ser que quienes trabajan menos de 40 horas a la semana (equivalente a 17\% de las observaciones) sean personas que alcanzaron un nivel salarial a partir del cual su oferta laboral más bien se reduce, aunque esta es una hipótesis poco plausible. Por último, el ingreso es creciente respecto al tamaño de la empresa en todos los modelos.

Las características sociodemográficas también se comportan según lo esperado. Quienes ejercen la jefatura del hogar reportan un salario, en promedio, $7 \%$ superior al de quienes no son jefes de hogar y la diferencia es mayor en la muestra masculina $(7,8 \%)$ que la femenina $(6,6 \%)$. Tener pareja también se asocia con un mayor salario, lo cual es consistente con una tendencia conocida en donde se premia a quienes se casan o forman hogares. Por el contrario, ser migrante se asocia con una caída en el salario de $5,1 \%$ en los hombres y $0,9 \%$ en las mujeres, respecto a las personas nacionales, mientras que las mujeres ganan, en promedio, 13,6\% menos que los hombres. Asimismo, el salario se reduce, en promedio, 2\% por cada menor de 6 años en el hogar. La zona de residencia también resulta significativa para explicar el ingreso: quienes viven en la región Central $y$ en zonas urbanas tienen acceso a mayores salarios que quienes habitan en otras regiones o en zonas rurales.

Finalmente, las estimaciones de $\operatorname{los} \lambda$ asociados al tipo de emparejamiento resultaron significativos en todos los casos. Efectivamente, entonces, la decisión del tipo de emparejamiento laboral y el salario se determinan simultáneamente, tal y como lo argumentan Dolton y Silles (2001) y Chevalier (2003).

El gráfico 5 resume las tasas de rendimiento asociadas a cada nivel de competencia relativo al primer nivel para el período observado, así como los rendimientos asociados a los tipos de emparejamiento. En primer lugar, se observa una caída de los rendimientos asociados con los hombres en el 2014 y que no se recupera sino hasta 2016. En contraste, los rendimientos de las mujeres se incrementan levemente para ese mismo año, aunque la variación observada en ellas no compensa la caída registrada en los rendimientos de los hombres. Esta brusca caída refleja el masivo cierre de empresas en 2014 y que afectó más fuertemente los rendimientos de la mano de obra más calificada. Durante ese año, solo dos empresas, Intel y Bank of America, despidieron a 1500 personas cada una. La prensa reportó un total de 5300 despidos y más de 20 empresas cerradas (Pérez, 2015 \& Fallas, 2015). Ante situaciones de despido como esta, es usual que algunas mujeres se reincorporen a la fuerza laboral para solventar la falta de recursos del hogar. Si quienes primero entran al mercado laboral son aquellas que tienen mejor posibilidad que su pareja de encontrar empleo, entonces es posible observar un aumento en sus rendimientos, tal y como lo muestra el gráfico 5 . 


\section{GRÁFICO 5 \\ RENDIMIENTO DE LOS NIVELES DE COMPETENCIAS Y PREMIO ASOCIADO AL TIPO DE EMPAREJAMIENTO LABORAL, SEGÚN GÉNERO. PERÍODO 2011-2017.}
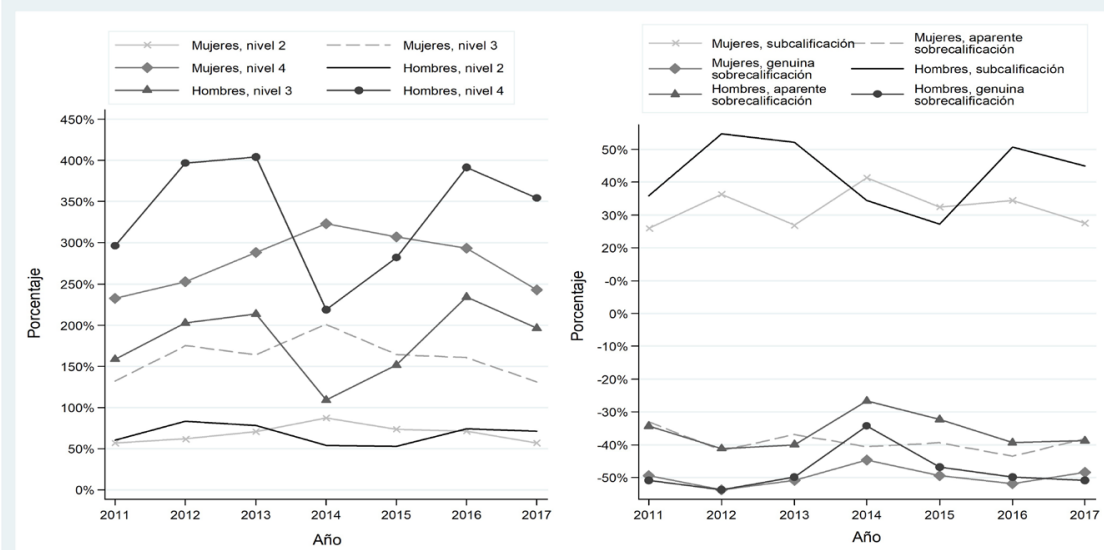

Fuente: Elaboración propia con datos de ENAHO.

Nota: Las líneas conectan los rendimientos obtenidos a partir de los coeficientes de las regresiones lineales para el logaritmo natural del salario real por hora controlando por características sociodemográficas, endogeneidad respecto al tipo de emparejamiento laboral, características del empleo y la empresa y características asociadas con el capital humano.

El gráfico también muestra que las personas con un nivel de competencia 2 ganan, en promedio, 60\% más que una persona de su mismo sexo con un nivel de competencia 1 y estos rendimientos parecen ser relativamente estables a lo largo del tiempo. Las mujeres, además, obtienen un rendimiento menor que el observado en la muestra masculina, con excepción de los años 2014 y 2015, probablemente por la hipótesis mencionada con anterioridad. Los rendimientos para las personas con un nivel de competencia 3 más que se duplican respecto a quienes poseen competencias en el nivel 1: las mujeres ganan, en promedio, un salario 140\% mayor al de las mujeres con un nivel de competencias 1; mientras que los hombres ganan $170 \%$ más que un hombre en la categoría de referencia. Para este nivel, la brecha entre los rendimientos de mujeres y hombres empieza a ensancharse en relación con lo observado en el nivel 2 y es aún más amplia si se observan los rendimientos asociados al nivel 4. En este caso, el salario de las mujeres es $252 \%$ más alto que el de aquellas con competencias del nivel 1, pero los hombres ganan 318\% más que los hombres en la categoría de referencia. Lo anterior reafirma la existencia de un techo de cristal para las mujeres. Finalmente, debe notarse que los rendimientos para 2017 disminuyen en todos los casos, reflejando las condiciones de desaceleración de la economía en ese momento.

En el segundo panel del gráfico 5 se muestran los rendimientos del tipo de emparejamiento relativos a quienes se encuentran adecuadamente empatadas. Con excepción del 2014-2015, los hombres mantuvieron un mayor premio por subcalificación que las mujeres, lo que, como se ha mencionado, sugiere que ellos son considerados más frecuentemente para ascensos y capacitaciones. Ahora bien, lo más interesante es que, las tasas de castigo por una aparente o genuina sobrecalificación son similares para ambos géneros, pero difieren a partir de la perturbación del 2014: el castigo se reduce para los hombres durante este tiempo y le toma como dos años volver a converger a una tasa similar a la de las mujeres. Es decir que, de alguna manera, la perturbación sobre los rendimientos de la educación para los hombres le fue compensada con un menor castigo por su sobrecalificación. 


\section{Población graduada de estudios superiores}

Debido a que la literatura acerca de la sobreeducación se centra en el estudio de la población con estudios superiores, en este apartado se presentan los resultados obtenidos para la submuestra de personas graduadas de estudios superiores (en adelante, personas graduadas), correspondientes a los niveles de competencia 3 y 4 . Esto permite analizar las variables en función de la inversión realizada en estudios superiores, medida a través de las áreas de estudio, en lugar de las ocupaciones.

\section{Logit multinomial para el tipo de emparejamiento: probabilidad de aparente sobrecalificación}

El cuadro 4 reporta los efectos marginales medios de las variables asociadas con el capital humano en la ecuación logit multinomial para el tipo de emparejamiento. Dado que el interés se centra sobre las decisiones de inversión y los emparejamientos observados, se omite el análisis de las variables sociodemográficas y aquellas relacionadas con la empresa utilizadas como control, ya que, en general, se comportan en forma similar a la muestra de la población mayor de edad.

En primer lugar, contar con estudios de posgrado reduce la probabilidad de una aparente sobrecalificación en 20 p.p. para las mujeres y 22,4 p.p. para los hombres. Además, todas las áreas de estudio se asocian con una mayor probabilidad de una aparente sobrecalificación en comparación con estudiar educación; pero estudios relacionados con servicios y las disciplinas sociales son las que se asocian con los mayores incrementos en dicha probabilidad. Además, merece resaltarse que las mujeres en áreas relacionadas con las ciencias, la salud y la matemática, ven incrementarse su probabilidad de una aparente sobrecalificación respecto a las mujeres en educación más que los hombres en estas mismas áreas en comparación con los hombres en educación, con la única excepción de ingeniería, arquitectura y manufactura. Lo anterior podría reflejar un comportamiento discriminatorio contra las mujeres en dichas áreas de estudio, tal que se les exige más calificaciones que a los hombres para desempeñarse en estos puestos. 
CUADRO 4

EFECTOS MARGINALES MEDIOS PARA LA LOGIT MULTINOMIAL SOBRE EL TIPO DE EMPAREJAMIENTO LABORAL DE LA POBLACIÓN CON ESTUDIOS SUPERIORES, POR GÉNERO (ERRORES ESTÁNDAR ROBUSTOS) PERÍODO: 2011-2017.

\begin{tabular}{|c|c|c|c|c|c|c|}
\hline & \multicolumn{2}{|c|}{ TOTAL } & \multicolumn{2}{|c|}{ MUJERES } & \multicolumn{2}{|c|}{ HOMBRES } \\
\hline & $\begin{array}{l}\text { Aparente } \\
\text { sobre- } \\
\text { calificación }\end{array}$ & $\begin{array}{c}\text { Sobre } \\
\text { calificación }\end{array}$ & $\begin{array}{l}\text { Aparente } \\
\text { sobre- } \\
\text { calificación }\end{array}$ & $\begin{array}{c}\text { Sobre } \\
\text { calificación }\end{array}$ & $\begin{array}{l}\text { Aparente } \\
\text { sobre- } \\
\text { calificación }\end{array}$ & $\begin{array}{c}\text { Sobre } \\
\text { calificación }\end{array}$ \\
\hline \multirow[t]{2}{*}{ Posee posgrado } & $-0,204 * * *$ & $-0,022 * * *$ & $-0,224 * * * *$ & $-0,031 * *$ & $-0,253 * * *$ & $-0,032 * *$ \\
\hline & $(0,008)$ & $(0,003)$ & $(0,020)$ & $(0,014)$ & $(0,021)$ & $(0,014)$ \\
\hline \multicolumn{7}{|c|}{ Área de estudio (base $=$ educación) } \\
\hline \multirow[t]{2}{*}{ Humanidades } & $0,132 * * *$ & $-0,006$ & $0,143 * * *$ & $-0,003$ & $0,125^{* * *}$ & $-0,034^{*}$ \\
\hline & $(0,021)$ & $(0,006)$ & $(0,026)$ & $(0,012)$ & $(0,035)$ & $(0,018)$ \\
\hline \multirow[t]{2}{*}{ Disciplinas sociales } & $0,257 * * *$ & $0,013 * * *$ & $0,252 * * *$ & $0,013 * *$ & $0,219 * * *$ & 0,002 \\
\hline & $(0,010)$ & $(0,004)$ & $(0,012)$ & $(0,006)$ & $(0,020)$ & $(0,008)$ \\
\hline \multirow{2}{*}{$\begin{array}{l}\text { Ciencias, matemática y } \\
\text { computación }\end{array}$} & $0,096^{* * *}$ & 0,000 & $0,152^{* * * *}$ & $-0,001$ & $0,064 * *$ & $-0,007$ \\
\hline & $(0,014)$ & $(0,005)$ & $(0,022)$ & $(0,011)$ & $(0,025)$ & $(0,009)$ \\
\hline \multirow{2}{*}{$\begin{array}{l}\text { Ingenierías, arquitectura y } \\
\text { manufacturas }\end{array}$} & $0,124 * * *$ & 0,007 & $0,099 * * *$ & 0,005 & $0,123 * * *$ & $-0,001$ \\
\hline & $(0,015)$ & $(0,006)$ & $(0,027)$ & $(0,010)$ & $(0,025)$ & $(0,009)$ \\
\hline \multirow{2}{*}{$\begin{array}{l}\text { Agricultura, pesca y ciencias } \\
\text { veterinarias }\end{array}$} & $0,115^{* * *}$ & $-0,007$ & $0,119 * * *$ & $-0,020$ & $0,097 * * *$ & $-0,022$ \\
\hline & $(0,023)$ & $(0,007)$ & $(0,046)$ & $(0,028)$ & $(0,033)$ & $(0,016)$ \\
\hline \multirow[t]{2}{*}{ Medicina y similares } & $0,097 * * *$ & 0,006 & $0,111 * * *$ & 0,010 & $0,080 * * *$ & $-0,013$ \\
\hline & $(0,014)$ & $(0,005)$ & $(0,018)$ & $(0,007)$ & $(0,030)$ & $(0,012)$ \\
\hline \multirow[t]{4}{*}{ Servicios } & $0,498 * * *$ & $0,038 * * *$ & $0,405^{* * *}$ & $0,029 * * *$ & $0,471 * * *$ & $0,030^{* * *}$ \\
\hline & $(0,027)$ & $(0,011)$ & $(0,030)$ & $(0,009)$ & $(0,038)$ & $(0,009)$ \\
\hline & $0,031 *$ & $-0,015^{*}$ & 0,030 & $-0,014^{*}$ & 0,036 & $-0,008$ \\
\hline & $(0,019)$ & $(0,008)$ & $(0,024)$ & $(0,007)$ & $(0,032)$ & $(0,009)$ \\
\hline Observaciones & 14489 & 14489 & 8002 & 8002 & 6487 & 6487 \\
\hline
\end{tabular}

Fuente: Elaboración propia con datos de ENAHO.

Nota: Se controla por tener un trabajo permanente, parcial, el tamaño de la empresa, trabajar en el sector privado, ser cabeza del hogar, tener pareja, ser migrante, ser mujer, la cantidad de menores de 6 años en el hogar, el tamaño del hogar, el número de personas desempleadas o inactivas en el hogar, la edad, edad al cuadrado y la región y zona de residencia.

\section{Logit multinomial para el tipo de emparejamiento: probabilidad de genuina sobrecalificación}

Los signos asociados a los coeficientes de los regresores para estimar la probabilidad de una genuina sobrecalificación son los mismos observados en la estimación de la probabilidad de una aparente sobrecalificación, pero se pierde la significancia para la mayoría de ellos. Las áreas de estudio dejan, por tanto, de ser relevantes, excepto por servicios: graduarse de esta área incrementa la probabilidad de una genuina sobrecalificación entre 2,9 y 3,9 p.p. 


\section{Ecuación de salarios}

El cuadro 5 presenta los resultados de la estimación del logaritmo natural del salario real por hora para la submuestra de personas graduadas. Como era de esperar, las personas con estudios de posgrado reciben un salario $34,2 \%$ superior a quienes tienen estudios de grado. Con excepción de las humanidades para el modelo agregado y los hombres, que presentan signos negativos, $y$ los servicios, que no son significativos, los coeficientes asociados a las áreas de estudio son positivos, i.e., el salario asociado a estas otras áreas de estudio es superior al de las personas graduadas de educación. Las carreras asociadas a la ciencia, matemática, computación, ingenierías, arquitectura y manufacturas se asocian con mayores salarios, aunque los rendimientos pueden variar considerablemente según el género. Por ejemplo, los hombres graduados en ciencia, matemática o computación ganan, en promedio, 22,8\% más que sus pares graduados de educación, mientras que las mujeres apenas ganan 19\% más que sus pares en la categoría de referencia. En cambio, en ingenierías, arquitectura y manufacturas el coeficiente asociado al modelo de las mujeres supera al del modelo masculino. Algo similar ocurre en las áreas relacionadas con salud: en agricultura, pesca y ciencias veterinarias las mujeres ganan $45 \%$ más que sus pares en educación, pero los hombres solo ganan $16 \%$ más; mientras que en salud el mayor rendimiento se observa en el modelo de la población masculina (24\%) y no el femenino (10\%). La experiencia, por su parte, se asocia en forma positiva $y$ decreciente con el salario y ser bilingüe incrementa el salario real por hora, en promedio, en $16 \%$.

Contar con algún tipo de sobrecalificación supone una considerable reducción del salario en comparación con quienes cuentan con el mismo nivel de competencias, pero se hallan en un trabajo adecuado para ellas. Así, la aparente sobrecalificación se asocia con una reducción salarial del $38 \%$, tanto para mujeres como hombres, y la genuina sobrecalificación, con una de 47,7\% para las mujeres y $51 \%$ para los hombres. Los empleos formales, medidos a través de la tenencia de seguro, suponen una mejora salarial en comparación con quienes no cuentan con él, al igual que tener un trabajo permanente respecto a uno temporal o contar con una jornada inferior a 40 horas semanales respecto a un trabajo de tiempo completo. El cambio en el salario de las mujeres asociado a la formalidad $(30,7 \%)$ y estabilidad $(11,4 \%)$ del empleo es, además, superior al observado en el modelo masculino. Por el contrario, quienes trabajan en el sector privado generan, en promedio, un ingreso 18,9\% inferior al de quienes laboran en el sector público. Para las mujeres, este cambio es aún mayor: las trabajadoras del sector privado ganan $21,3 \%$ menos que sus pares en el sector público. Además, el tamaño de la empresa se asocia positiva y crecientemente al salario.

En cuanto a las características sociodemográficas, ser cabeza del hogar se asocia con un ingreso 9,5\% adicional en el caso de las mujeres y 14,1\% en los hombres. Similarmente, tener pareja se relaciona con un incremento de alrededor del $10 \%$ en el ingreso, con un mayor premio observado en las mujeres (11,7\%) que en los hombres (7,3\%). Los coeficientes asociados a ser migrante no son significativos al nivel de confianza estándar, lo cual supone una diferencia respecto a la población total, en donde las personas migrantes si eran castigadas en su salario. Las mujeres, en cambio, reportan un salario 6,8\% inferior al de los hombres y, en general, residir en la región Central o en zonas urbanas se asocia con mayores opciones salariales. Finalmente, los lambdas asociados a la aparente y genuina sobrecalificación únicamente resultan significativos para las mujeres al nivel de confianza estándar, es decir, solo en este modelo se observa endogeneidad entre la determinación del salario y el tipo de emparejamiento laboral. 
CUADRO 5

ECUACIÓN PARA EL LOGARITMO NATURAL DEL SALARIO REAL POR HORA DE LA POBLACIÓN CON ESTUDIOS SUPERIORES, POR GÉNERO (ERRORES ESTÁNDAR ROBUSTOS) PERÍODO: 2011-2017.

\begin{tabular}{|c|c|c|c|}
\hline & Total & Mujeres & Hombres \\
\hline \multirow[t]{2}{*}{ Posee posgrado } & $0,294 * * *$ & $0,309^{* * *}$ & $0,308^{* * *}$ \\
\hline & $(0,018)$ & $(0,023)$ & $(0,035)$ \\
\hline \multicolumn{4}{|l|}{ Área de estudio (base $=$ educación) } \\
\hline \multirow[t]{2}{*}{ Humanidades } & $-0,030$ & 0,054 & $-0,127 * * * *$ \\
\hline & $(0,031)$ & $(0,037)$ & $(0,051)$ \\
\hline \multirow[t]{2}{*}{ Disciplinas sociales } & $0,182^{* * *}$ & $0,169 * * *$ & $0,169 * * *$ \\
\hline & $(0,015)$ & $(0,019)$ & $(0,027)$ \\
\hline \multirow[t]{2}{*}{ Ciencias, matemática y computación } & $0,205^{* * *}$ & $0,174 * * *$ & $0,205^{* * *}$ \\
\hline & $(0,018)$ & $(0,027)$ & $(0,025)$ \\
\hline \multirow[t]{2}{*}{ Ingenierías, arquitectura y manufacturas } & $0,202^{* * *}$ & $0,257 * * *$ & $0,174 * * *$ \\
\hline & $(0,020)$ & $(0,036)$ & $(0,028)$ \\
\hline \multirow[t]{2}{*}{ Agricultura, pesca y ciencias veterinarias } & $0,188^{* * *}$ & $0,373^{* * *}$ & $0,150 * * *$ \\
\hline & $(0,031)$ & $(0,062)$ & $(0,038)$ \\
\hline \multirow[t]{2}{*}{ Medicina y similares } & $0,142^{* * *}$ & $0,100^{* * *}$ & $0,216 * * *$ \\
\hline & $(0,019)$ & $(0,023)$ & $(0,033)$ \\
\hline \multirow[t]{2}{*}{ Servicios } & $0,082 * *$ & 0,066 & 0,077 \\
\hline & $(0,033)$ & $(0,042)$ & $(0,055)$ \\
\hline \multirow[t]{2}{*}{ Años de experiencia laboral } & $0,019 * * *$ & $0,021 * * *$ & $0,019 * * *$ \\
\hline & $(0,002)$ & $(0,002)$ & $(0,003)$ \\
\hline \multirow[t]{2}{*}{ Experiencia ${ }^{2}$} & $-0,000 * * *$ & $-0,000^{* * *}$ & $-0,000 * * *$ \\
\hline & $(0,000)$ & $(0,000)$ & $(0,000)$ \\
\hline \multicolumn{4}{|l|}{ Calificación (base = calificación) } \\
\hline \multirow[t]{2}{*}{ Aparente sobrecalificación } & $-0,492 * * *$ & $-0,484 * * *$ & $-0,499 * * *$ \\
\hline & $(0,011)$ & $(0,015)$ & $(0,016)$ \\
\hline \multirow[t]{2}{*}{ Genuina sobrecalificación } & $-0,680 * * *$ & $-0,649 * * *$ & $-0,714 * * *$ \\
\hline & $(0,026)$ & $(0,034)$ & $(0,040)$ \\
\hline \multirow[t]{2}{*}{ Es bilingüe } & $0,148 * * *$ & $0,143^{* * *}$ & $0,146^{* * * *}$ \\
\hline & $(0,010)$ & $(0,014)$ & $(0,015)$ \\
\hline \multirow[t]{2}{*}{ Cuenta con seguro } & $0,230 * * *$ & $0,268 * * *$ & $0,196 * * *$ \\
\hline & $(0,026)$ & $(0,035)$ & $(0,038)$ \\
\hline \multirow[t]{2}{*}{ Trabajo permanente } & $0,098 * * *$ & $0,108^{* * *}$ & 0,074 \\
\hline & $(0,026)$ & $(0,031)$ & $(0,046)$ \\
\hline \multirow[t]{2}{*}{ Trabajo parcial } & $0,321 * * *$ & $0,318^{* * * *}$ & $0,342 * * *$ \\
\hline & $(0,014)$ & $(0,016)$ & $(0,027)$ \\
\hline \multirow[t]{2}{*}{ Trabaja en el sector privado } & $-0,210 * * *$ & $-0,240 * * *$ & $-0,184 * * *$ \\
\hline & $(0,011)$ & $(0,015)$ & $(0,018)$ \\
\hline
\end{tabular}




\begin{tabular}{|c|c|c|c|}
\hline & Total & Mujeres & Hombres \\
\hline \multicolumn{4}{|l|}{ Tamaño de la empresa $($ base $=$ microempresa $)$} \\
\hline \multirow[t]{2}{*}{ Pequeña } & $0,080^{* * *}$ & $0,072^{* * * *}$ & $0,102^{* * * *}$ \\
\hline & $(0,016)$ & $(0,020)$ & $(0,025)$ \\
\hline \multirow[t]{2}{*}{ Mediana } & $0,125^{* * *}$ & $0,128^{* * * *}$ & $0,132^{* * *}$ \\
\hline & $(0,015)$ & $(0,019)$ & $(0,025)$ \\
\hline \multirow[t]{2}{*}{ Grande } & $0,219 * * *$ & $0,228^{* * * *}$ & $0,217^{* * * *}$ \\
\hline & $(0,015)$ & $(0,020)$ & $(0,024)$ \\
\hline \multirow[t]{2}{*}{ Es cabeza del hogar } & $0,097 * * *$ & $0,091 * * *$ & $0,132 * * *$ \\
\hline & $(0,010)$ & $(0,013)$ & $(0,020)$ \\
\hline \multirow[t]{2}{*}{ Tiene pareja } & $0,102^{* * *}$ & $0,111^{* * * *}$ & $0,070^{* * *}$ \\
\hline & $(0,010)$ & $(0,012)$ & $(0,019)$ \\
\hline \multirow[t]{2}{*}{ Es migrante } & $-0,011$ & $-0,022$ & $-0,014$ \\
\hline & $(0,024)$ & $(0,031)$ & $(0,036)$ \\
\hline \multirow[t]{2}{*}{ Es mujer } & $-0,070^{* * *}$ & & \\
\hline & $(0,009)$ & & \\
\hline \multicolumn{4}{|l|}{ Región (referencia = Central) } \\
\hline \multirow[t]{2}{*}{ Chorotega } & $-0,133 * * *$ & $-0,090^{* * *}$ & $-0,196 * * *$ \\
\hline & $(0,018)$ & $(0,021)$ & $(0,031)$ \\
\hline \multirow[t]{2}{*}{ Pacífico Central } & $-0,121 * * *$ & $-0,105^{* * * *}$ & $-0,141 * * *$ \\
\hline & $(0,022)$ & $(0,029)$ & $(0,034)$ \\
\hline \multirow[t]{2}{*}{ Brunca } & $-0,032^{* * *}$ & $-0,019$ & $-0,054 * *$ \\
\hline & $(0,016)$ & $(0,020)$ & $(0,025)$ \\
\hline \multirow[t]{2}{*}{ Huetar Atlántica } & $-0,137 * * *$ & $-0,107 * * *$ & $-0,167 * * *$ \\
\hline & $(0,020)$ & $(0,026)$ & $(0,034)$ \\
\hline \multirow[t]{2}{*}{ Huetar Norte } & $-0,005$ & 0,033 & $-0,047^{*}$ \\
\hline & $(0,016)$ & $(0,020)$ & $(0,025)$ \\
\hline \multirow[t]{2}{*}{ Zona urbana } & $0,043^{* * *}$ & $0,026^{*}$ & $0,064 * * *$ \\
\hline & $(0,010)$ & $(0,014)$ & $(0,016)$ \\
\hline \multirow[t]{2}{*}{ asociado a la aparente sobrecalificación } & $0,006^{*}$ & $0,013^{* * * *}$ & 0,008 \\
\hline & $(0,004)$ & $(0,004)$ & $(0,009)$ \\
\hline \multirow[t]{2}{*}{ asociado a la genuina sobrecalificación } & 0,001 & $0,001^{* *}$ & 0,000 \\
\hline & $(0,000)$ & $(0,001)$ & $(0,001)$ \\
\hline \multirow[t]{2}{*}{ Constante } & $7,642^{* * * *}$ & $7,563^{* * *} *$ & $7,685^{* * * *}$ \\
\hline & $(0,043)$ & $(0,053)$ & $(0,076)$ \\
\hline Observaciones & 14488 & 8002 & 6486 \\
\hline $\mathrm{F}$ & 330,00 & 194,07 & 148,92 \\
\hline $\mathrm{R} 2$ & 0,415 & 0,417 & 0,411 \\
\hline $\mathrm{R} 2$ ajustado & 0,414 & 0,415 & 0,408 \\
\hline
\end{tabular}

Fuente: Elaboración propia con datos de ENAHO. 


\section{DISCUSIÓN Y CONCLUSIONES}

En este estudio se estimaron las tasas de subcalificación y sobrecalificación para la población mayor de edad ocupada en Costa Rica. Los resultados muestran que, mediante los métodos estadísticos se encuentran tasas dentro de los rangos observados en otros países, pero esto puede deberse al mismo método de estimación que consiste en identificar las colas de una distribución. En contraste, el método objetivo genera una tasa de subcalificación de 28,9\%, consistente con el hecho de que $70 \%$ de la población mayor de edad tiene un nivel educativo inferior a secundaria completa y $72 \%$ de la población ocupada labora en puestos que requieren niveles de competencia 1 o 2. Este indicador pone en evidencia la falta de capital humano relativo a los estándares internacionales sobre los requerimientos de competencias para las distintas ocupaciones y constituye una alerta sobre la necesidad de capacitar a la población en edad de trabajar, para que desarrolle competencias específicas, en especial, habilidades técnicas de nivel intermedio, pues este nivel es prácticamente inexistente en la oferta laboral. De hecho, la mayor tasa de subcalificación $(65,9 \%)$ se observa en ocupaciones técnicas y profesionales de nivel intermedio. Asimismo, las ocupaciones que exigen niveles de competencia 2 y 3 se asocian con una mayor probabilidad de subcalificación. Lo anterior caracteriza un mercado laboral dual, en donde la mayor parte de la población, al carecer de habilidades complejas, deberá limitarse a puestos de baja calificación; mientras que el pequeño porcentaje con estudios superiores puede asegurarse contra el desempleo, la subcalificación y obtener rendimientos sobre sus habilidades considerables debido a la escasez de mano de obra capacitada.

Así, la sobrecalificación no representa un problema en el caso costarricense. Si bien 14,2\% de la población ocupada está sobrecalificada para su puesto de trabajo, esto es solo en apariencia, pues el porcentaje de personas insatisfechas con su trabajo por un desempate de habilidades y requerimientos laborales es marginal $(0,8 \%)$. La diferencia entre la tasa de aparente y genuina sobrecalificación, sin embargo, puede constituir otra alerta sobre la calidad del sistema educativo costarricense, i.e., las personas podrían no estar desarrollando las competencias que se supone se asocian con su nivel educativo y, por tanto, se sienten satisfechas en puestos que requieren menos de ellas.

Los malos emparejamientos, además, parecen estar influidos por los roles tradicionales de género: las mujeres con pareja o menores en edad preescolar reportan una menor probabilidad de subcalificación, posiblemente porque prefieren retirarse del mercado laboral. Además, contar con pareja también incrementa la probabilidad de sobrecalificación de las mujeres, en concordancia con la tesis de que, al limitar su búsqueda de trabajo al de su pareja, optan por trabajos inferiores a sus habilidades. La edad de las mujeres más bien se relaciona con el tipo de emparejamiento en forma inversa a la esperada, de forma que la probabilidad de subcalificación (sobrecalificación) aumenta (disminuye) con la edad, posiblemente por autoselección: aquellas con mayor arraigo al mercado laboral tienen mayores posibilidades de ascenso relativas a su nivel de competencias. En cambio, los hombres con pareja y menores en el hogar ven incrementada su probabilidad de un mal emparejamiento, al asumir el rol de proveedor en el hogar. Asimismo, las mujeres reportan una menor probabilidad de subcalificación y mayor probabilidad de sobrecalificación que los hombres.

Dentro de las características del empleo, el mayor tamaño de las empresas y el sector público favorecen mejores emparejamientos, sobre todo contribuyendo a disminuir la subcalificación. En cuanto a la población graduada, estudiar áreas relacionadas con servicios o disciplinas sociales incrementa en mayor medida la probabilidad de sobrecalificación y las mujeres ven incrementada su probabilidad de sobrecalificación en áreas relacionadas con las ciencias, la salud y la matemática, lo que refuerza la segregación de áreas de estudio, pues parece que se les castiga por incursionar en carreras tradicionalmente masculinas al exigirles más requisitos de los necesarios. 
Las ecuaciones de salario muestran que los rendimientos asociados al nivel de competencia son positivos y crecientes, pero que existe evidencia de la existencia de un techo de cristal, pues los rendimientos asociados a los niveles de competencia son superiores para los hombres y las diferencias se acrecientan a lo largo de la escala de competencias. Adicionalmente, el premio por subcalificación de 30,5\% es superior al reportado en otros países, reflejando la escasez de mano de obra calificada en Costa Rica, lo mismo podría argumentarse sobre los considerables castigos a la aparente y genuina sobrecalificación. Uno de los resultados más interesantes, sin embargo, es que durante perturbaciones del empleo como la observada en el 2014, el castigo a la sobrecalificación de los hombres se ajusta para compensar los efectos negativos de la perturbación. Para las personas graduadas, las tasas de castigo por sobrecalificación son superiores a las observadas en la población mayor de edad.

En general, las conclusiones de este estudio advierten sobre la necesidad de planear adecuadamente las políticas de inversión en capital humano, pues los costos asociados a estas malas decisiones no se reflejan únicamente en el costo de la inversión, sino también en sus retornos esperados. Asimismo, se evidencia la necesidad de diseñar una política educativa que abarque, no solo la educación formal para el desarrollo de competencias más complejas, sino un proceso masivo de capacitación a la población que actualmente tiene edad de trabajar y que, en su gran mayoría, constituye mano de obra no calificada. No hacerlo supone perpetuar una política de crecimiento basada en la mano de obra no calificada y de baja productividad para la mayoría de la población.

\section{AGRADECIMIENTOS}

Se agradece a María José Sauma y Víctor Picado por su labor asistiendo en etiquetar las bases de datos.

\section{REFERENCIAS}

Alba-Ramírez, A. (1993). Mismatch in the Spanish labor market: overeducation? The Journal of Human Resources, 28(2), 259-278. https://doi.org/10.2307/146203

Allen, J., \& Van der Velden, R. (2001). Educational mismatches versus skill mismatches: effects on wages, job satisfaction, and on-the-job search. Oxford Economic Papers, 53(3), 434-452. https://doi.org/10.1093/oep/53.3.434

Alpin, C., Shackleton, J.R. \& Walsh, S. (1998). Over- and undereducation in the UK graduate labour market. Studies in Higher Education, 23(1), 17-34. https://doi.org/10.1080/03075079812331380462

Battu, H., Belfield, C. R., \& Sloane, P. J. (1999). Overeducation among graduates: a cohort view. Education Economics, 7(1), 21-38. https://doi.org/10.1080/09645299900000002

Becker, G.S. (1962). Investment in human capital: a theoretical analysis. Journal of Political Economy, 70(5), 9-49. https://doi.org/10.1086/258724

Becker, G. (1993). Human capital. A theoretical and empirical analysis with special reference to education. Chicago: The University of Chicago Press and National Bureau of Economic Research.

Bennell, P. (1996). General vs technical secondary education in developing countries: a review of the rates of return evidence. The Journal of Development Studies, 33(2), 230-247. https://doi.org/10.1080/00220389608422464

Blau, F.D. (1999). Women's economic well-being, 1970-1995: Indicators and trends. Focus, 20(1), 4-10. 
Blau, F.D. y Kahn, L.M. (2007). The gender pay gap: have women gone as far as they can? Academy of Management Perspectives, 21(1), 7-23. https://doi.org/10.5465/amp.2007.24286161

Boll, C., Leppin, J., Rossen, A. \& Wolf, A. (2016). Overeducation - new evidence for 25 European countries, HWWI Research Paper 173, Hamburg Institute of International Economics (HWWI).

Chevalier, A. (2003). Measuring over-education. Economica, 70(279), 509-531. https://doi.org/10.1111/1468-0335.t01-1-00296

Chevalier, A. (2011). Subject choice and earnings of UK graduates. Economics of Education Review, 30(6), 1187-1201. https://doi.org/10.1016/j.econedurev.2011.04.007

Chevalier, A., \& Lindley, J. (2009). Overeducation and the skills of UK graduates. Journal of the Royal Statistical Society: Series A (Statistics in Society), 172(2), 307-337. https://doi.org/10.1111/j.1467-985X.2008.00578.x

de Grip, A., Bosma, H., Willems, D., \& van Boxtel, M. (2008). Job-worker mismatch and cognitive decline. Oxford Economic Papers, 60(2), 237-253. https://doi.org/10.1093/oep/gpm023

Dolton, P. \& Silles, M. (2001). Over-education in the graduate labour market: some evidence from alumni data. London: Centre for the economics of education, London School of Economics and Political Science.

Eichhorst, W., Rodríguez-Planas, N., Schmidl, R., \& Zimmermann, K. F. (2015). A road map to vocational education and training in industrialized countries. ILR Review, 68(2), 314-337. https://doi.org/10.1177/0019793914564963

European Commission, Directorate General Education and Culture (2005). The returns to various types of investments in education and training. Disponible en: https://londoneconomics. co.uk/wp-content/uploads/2011/09/82-Study-on-the-returns-to-various-types-of-investmentin-education-and-training.pdf.

Fallas, C. (5 de agosto de 2015). Al menos 22 empresas han anunciado cierres y despidos en los últimos 19 meses. El Financiero. Disponible en: https://www.elfinancierocr.com/ negocios/al-menos-22-empresas-han-anunciado-cierres-y-despidos-en-los-ultimos-19-meses/ VMB4KD7CDRBJ7BJNB5ODZH7BSM/story/

Farooq, S. (2011). Mismatch between education and occupation: a case study of Pakistani graduates. The Pakistan Development Review, 50(4II), 531-553. https://doi.org/10.30541/v50i4IIpp

Farooq, S. (2015). Job mismatches in Pakistan: is there some wage penalty to graduates? The Pakistan Development Review, 54(2), 147-164. https://doi.org/10.30541/v54i2pp.147-164

Fernández, C. and Ortega, C. (2006). Labour market assimilation of immigrants in Spain: employment at the expense of bad-job matches?. (IESE Working Paper 644), University of Navarra.

Groot, W., \& van den Brink, H.M. (2000). Overeducation in the labor market: A meta-analysis. Economics of Education Review, 19(2), 149-158. https://doi.org/10.1016/S0272-7757(99)00057-6

Heckman, J.J. (1976). The common structure of statistical models of truncation, sample selection and limited dependent variables and a simple estimator for such models. Annals of Economic and Social Measurement, 5(4), 475-492.

Instituto Nacional de Estadística y Censos (2014). Clasificación de Ocupaciones de Costa Rica COCR2011. San José: INEC.

Instituto Nacional de Estadística y Censos (2016). Encuesta Nacional de Hogares, julio 2016: resultados generales. San José: INEC.

Instituto Nacional de Estadística y Censos (2018). Encuesta Nacional de Hogares 2011-2017. San José: INEC.

Instituto Nacional de Estadística y Censos (2019). Encuesta Continua de Empleo al tercer trimestre de 2019. Resultados generales. San José: INEC. 
International Labour Organization (2012a). International Standard Classification of Occupations, Geneva: ILO.

International Labour Organization (2012b). International Standard Classification of Occupations, Annex 1. Geneva: ILO.

International Labour Organization (2014). Skills mismatch in Europe. Statistics Briefing, Geneva: ILO.

Jauhiainen, S. (2011). Overeducation in the Finnish regional labour markets: Overeducation and regional labour markets. Papers in Regional Science, 90(3), 573-588. https://doi.org/10.1111/j.1435-5957.2010.00334.x

Jovanovic, B. (1979). Job matching and the theory of turnover. Journal of Political Economy, 87(5, Part 1), 972-990. https://doi.org/10.1086/260808

Kucel, A. (2011). Literature survey of the incidence of over-education: a sociological approach. Revista Española de Investigaciones Sociológicas, (134), 125-142.

Marqués Perales, I. \& Gil-Hernández, C.J. (2015). Origen social y sobreeducación en los universitarios españoles: ¿es meritocrático el acceso a la clase de servicio? Revista Española de Investigaciones Sociológicas, (150), 89-112. https://doi.org/10.5477/cis/reis.150.89

Mavromaras, K., McGuinness, S., \& Fok, Y. K. (2009). Assessing the incidence and wage effects of over-skilling in the Australian labour market. Economic Record, 85(268), 60-72. https://doi.org/10.1111/j.1475-4932.2008.00529.x

McMillen, D.P., Seaman, P.T. \& Singell Jr., L.D. (2007). A mismatch made in heaven: a hedonic analysis of overeducation and undereducation. Southern Economic Journal, 73(4), 901-930. https://doi.org/10.2307/20111934

Mincer, J. (1974). Schooling, experience and earnings. New York: Columbia University Press.

Montenegro, C.E. \& Patrinos, H.A. (2013). Returns to schooling around the world. (Background papers for the World Development Report 2013).

Mumford, K. y Smith, P. N. (2009). What determines the part-time and gender earning gaps in Britain: evidence from the workplace. Oxford Economic Papers, 61(suppl_1), i56-i75. https://doi.org/10.1093/oep/gpn041

Ortiz, L. \& Kucel, A. (2008). Do fields of study matter for over-education? The cases of Spain and Germany. International Journal of Comparative Sociology, 49(4-5), 305-327. https://doi.org/10.1177/0020715208093079

Pérez, D. (9 de enero de 2015). Salida de Wendy's eleva a más de 5.300 los desempleados por cierre de empresas. La Prensa Libre. Disponible en: http://www.laprensalibre.cr/Noticias/ detalle/8304--salida-de-wendys-eleva-a-mas-de-5300-los-desempleados-por-cierre-deempresas

Programa Estado de la Nación (2019). Informe Estado de la Nación 2019. San José: Servicios Gráficos AC 2019.

Quintini, G. (2011). Over-qualified or under-skilled: a review of existing literature. OECD Social, Employoment and Migration Working Papers, 121, Organisation for Economic Cooperation and Development. https://doi.org/10.1787/5kg58j9d7b6d-en

Sattinger, M. (1993). Assignment models of the distribution of earnings. Journal of Economic Literature, 31(2), 831-880.

Schleicher, A. (2017). Análisis de la OCDE acerca de las políticas nacionales para educación: la educación en Costa Rica. Organización para la Cooperación y el Desarrollo Económico. Disponible en: https:/www.oecd.org/edu/school/La-Educacion-en-Costa-Rica-ResumenEjecutivo.pdf

Schultz, T.P. (1974). The economics of the family: marriage, children and human capital. National Bureau of Economic Research.Thurow, L.C. (1975) Generating Inequality. New York: Basic Books. 
Sicherman, N. (1991). "Overeducation" in the labor market. Journal of Labor Economics, 9(2), 101-122. https://doi.org/10.1086/298261

Sicherman, N. \& Galor, O. (1990). A theory of career mobility. Journal of Political Economy, 98(1), 169-192. https://doi.org/10.1086/261674

Sloane, P.J., Battu, H. \& Seaman, P.T. (1999). Overeducation, undereducation and the British labour market. Applied Economics, 31(11), 1437-1453. https://doi.org/10.1080/000368499323319

Spence, M. (1973). Job market signaling. The Quarterly Journal of Economics, 87(3), 355-374. https://doi.org/10.2307/1882010

Sutherland, J. (2012). Qualifications mismatch and skills mismatch. Education + Training, 54(7), 619-632. https://doi.org/10.1108/00400911211265666

Thurow, L.C. (1975). Generating inequality. New York: Basic Books.

Verdugo, R.R. \& Verdugo, N.T. (1988) Overeducation and the earnings of black, hispanic, and

white male workers. Sociological Perspectives, 31(2), 190-212. https://doi.org/10.2307/1389082

Verhaest, D. \& Omey, E. (2006). The impact of overeducation and its measurement. Social Indicators Research, 77(3), 419-448. https://doi.org/10.1007/s11205-005-4276-6 